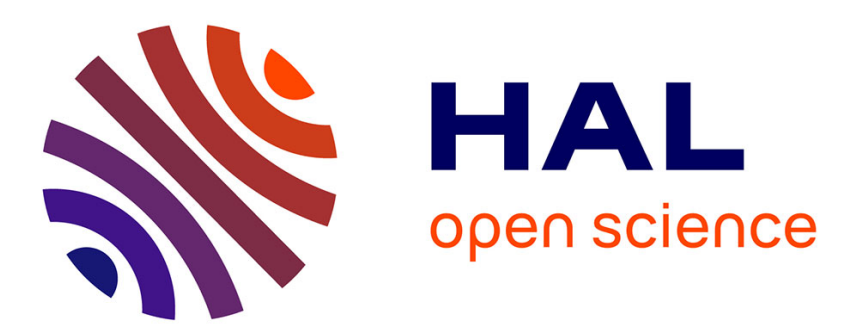

\title{
Sémantique du passé composé en français moderne et exploration des rapports passé composé / passé simple dans un corpus de moyen français
}

Denis Apothéloz

\section{- To cite this version:}

Denis Apothéloz. Sémantique du passé composé en français moderne et exploration des rapports passé composé / passé simple dans un corpus de moyen français. Pierre-Don Giancarli, Marc Fryd. Aoristes et parfaits en français, latin, corse, estonien et polonais, 28, Brill; Rodopi, pp.199-246, 2016, Cahiers Chronos, 978-90-04-31338-05. 10.1163/9789004313408_011. hal-01132723

\section{HAL Id: hal-01132723 \\ https://hal.science/hal-01132723}

Submitted on 19 Mar 2020

HAL is a multi-disciplinary open access archive for the deposit and dissemination of scientific research documents, whether they are published or not. The documents may come from teaching and research institutions in France or abroad, or from public or private research centers.
L'archive ouverte pluridisciplinaire HAL, est destinée au dépôt et à la diffusion de documents scientifiques de niveau recherche, publiés ou non, émanant des établissements d'enseignement et de recherche français ou étrangers, des laboratoires publics ou privés. 
Article paru dans :

Pierre-Don Giancarli, Marc Fryd (éds):

Aoristes et parfaits en français, latin, corse, estonien et polonais. (= Cahiers Chronos 28) Leiden / Boston : Brill / Rodopi, 2016, pp. 199-246.

\title{
Sémantique du passé composé en français moderne et exploration des rapports passé composé / passé simple dans un corpus de moyen français ${ }^{1}$
}

\author{
Denis APOTHÉLOZ \\ Université de Lorraine \& laboratoire ATILF (CNRS \& UL)
}

\begin{abstract}
This article aims to offer new insights regarding the analysis and the history of the French passé composé. In particular, it re-examines the claim that this tense has become an aorist. The author begins by stating his vision of the passé composé's semantics, paying particular attention to its resultative property. The core element is the concept of factuality. Three types of factuality in discourse are distinguished, one of them being the experiential type (i.e. the "experiential perfect"). The final part of the article is an empirical study where the uses of passé simple and passé composé in P. de Commynes' Mémoires (Middle French) are compared. The competition between the two tenses in certain types of factual utterances is highlighted, as well as how set phrases have helped keep the passé simple in use.
\end{abstract}

Keywords: French passé composé and passé simple, experiential perfect, factual sentence, resultativity, superlativity.

\section{Introduction}

Le but du présent article est d'apporter quelques éléments d'analyse et de réflexion concernant l'histoire du passé composé (désormais PC). L'un des points que nous examinerons est la thèse selon laquelle le PC est un temps verbal en voie d'aoristisation, thèse qui semble désormais faire l'unanimité parmi les linguistes. Nous commencerons par rappeler la «vulgate » en ce qui concerne l'histoire du PC (section 2). Puis, nous exposerons une conception quelque peu différente de la sémantique de ce temps verbal

Merci à M. Nowakowska, M. Fryd, P.D. Giancarli et aux relecteurs anonymes, dont les remarques et suggestions ont permis d'améliorer considérablement la clarté de cet article. Merci également à $\mathrm{B}$. Combettes de ses précieux conseils concernant la traduction des exemples d'ancien et de moyen français. 
(section 3). Un élément central de notre argumentation est l'introduction de la notion de «fait» et d'énoncé factuel. Cette notion nous amènera à proposer une analyse du PC différente de celle qu'on rencontre habituellement dans la littérature, notamment en ce qui concerne le contenu du composant résultatif. Elle nous conduira également à distinguer trois variantes d'énoncés factuels, parmi lesquels figurent les énoncés comportant un parfait d'expérience. Nous reviendrons également sur ce qu'il convient d'appeler «narration » et sur ce qui oppose le passé simple (PS) au PC à cet égard (section 4).

Selon Martin (1971), l'évolution du PC marque un tournant décisif durant la période du moyen français. En effet, bien qu'il soit attesté comme temps du passé dès la période de l'ancien français, c'est surtout vers la fin du $\mathrm{XV}^{\mathrm{e}}$ s., selon cet auteur, que ce temps verbal paraît développer des emplois le rapprochant d'un aoriste («prétérit», dans les termes de Martin). C'est donc tout naturellement vers cette époque que nous avons choisi de nous tourner pour la partie proprement empirique de ce travail (section 5). Munis des instruments d'analyse exposés dans les sections précédentes, nous y présentons les résultats d'une première exploration des emplois du $\mathrm{PC}$ et du PS dans les Mémoires de Philippe de Commynes (1447-1511). Un point qui a tout particulièrement attiré notre attention est la concurrence qui parait se jouer entre ces deux temps verbaux dans certains énoncés factuels, et l'effet conservateur que produisent sur le PS certaines formulations stéréotypées, " phraséologiques », de la superlativité.

A l'origine, notre projet était de mener une étude diachronique de la valeur de parfait d'expérience. L'hypothèse était qu'une telle étude apporterait des données nouvelles et une perspective inédite, aussi bien sur l'histoire du PC que sur l'histoire de la relation PC-PS. Ce projet a subi ensuite divers réajustements. Il est en effet progressivement apparu que le type particulier de résultativité qui caractérise le parfait d'expérience était de nature à remettre en cause toute l'analyse du PC en général. Ce déplacement $\mathrm{du}$ projet initial explique que cet article puisse donner l'impression de poursuivre deux lièvres à la fois: d'une part, repenser la description sémantique du $\mathrm{PC}$ et, à moyen terme, des temps composés; d'autre part, étudier le fonctionnement du PC et l'opposition PC/PS en moyen français.

\section{Histoire du passé composé}

Les linguistes s'accordent généralement pour considérer qu'à l'origine du PC se trouve une construction attributive dans laquelle un participe adjectival a une fonction d'attribut d'un complément d'objet du verbe avoir (cf. déjà Brunot \& Bruneau 1949). L'origine du PC serait donc dans des constructions analogues à celles réunies en (1). 
Est-ce que vous avez votre ceinture attachée?

En principe, avoir est ici un verbe lexical ${ }^{2}$; il régit un complément (mon pantalon, les yeux, etc.), et l'agent responsable de l'état désigné par le participe adjectival n'est pas astreint à coïncider avec le référent du sujet du verbe avoir (l'agent de la déchirure du pantalon n'est pas nécessairement la personne désignée par «je », etc.). On a donc à cette étape une construction syntaxique, et non pas un temps verbal. Cette construction est qualifiée parfois de "résultative », et le participe adjectival y est décrit comme un passif statif ${ }^{3}$.

Pour qu'il y ait temps verbal, il faut attendre que certains emplois de cette construction subissent deux modifications importantes, dont l'aboutissement est le suivant :

(i) le référent du sujet du verbe avoir coïncide avec l'agent de la situation décrite par le participe adjectival ;

(ii) le verbe avoir subit une érosion sémantique et perd son sens de «possession ».

Cette double transformation, que Timberlake (1977) a attribuée à un mécanisme de réanalyse, a pour conséquence de déplacer le centre de gravité de la construction : avoir perd son statut de verbe lexical au profit du verbe que représente le participe, et n'est plus qu'un verbe «auxiliaire»; corrélativement, le sujet grammatical et le complément quittent le champ rectionnel de avoir pour celui du verbe représenté par le participe. Ces modifications s'accompagnent, du point de vue de l'ordre des mots, d'un rapprochement entre l'auxiliaire et le participe.

Ce nouveau grammème de temps verbal a pour fonction principale de signifier, et donc de désigner, un état présent, valide au moment de l'énonciation (désormais $\mathrm{T}_{0}$ ), tout en indiquant que cet état est consécutif à un événement passé. Sa valeur est donc celle d'un "présent résultatif», ou

2 «En principe», parce que dans ces formulations, du moins dans certaines de leurs énonciations, avoir pourrait être considéré comme un auxiliaire de construction présentative (j'ai mon pantalon déchiré équivalant alors à il y a mon pantalon qui est déchiré). Voir sur ce point Lambrecht (1994) et ses exemples de « sentence-focus structures ».

3 Dans certains cas, la construction s'est lexicalisée : avoir et le SN qui le suit forment alors, par figement, une expression verbale composée. C'est ce qu'on observe dans l'expression avoir l'air, dans il a l'air surpris. Il s'agit là, évidemment, d'un tout autre devenir de la construction attributive. 
accompli. Nous qualifierons de «résultatif» cet emploi du PC. Selon Marchello-Nizia (1999), cette étape est réalisée au V $\mathrm{e}^{\mathrm{e}} \mathrm{s}$. déjà, donc bien avant l'existence du « français ». En ancien français, soit quatre siècles plus tard, ce type de PC est tout à fait ordinaire. Il est pratiquement toujours associé à la construction d'un arrière-plan de la narration. On le rencontre typiquement dans deux environnements :

- d'une part, dans des séquences au présent de narration, comme dans (2). Il sert alors, comme en français moderne, à décrire un état résultant incluant temporellement les situations décrites au présent de narration. Cet état résultant constitue ainsi le cadre dans lequel prennent place les événements narratifs.

Rollant AD MIS l'olifan a sa buche,

EMPEINT le ben, par grant vertut le SUNET. (Ch. de Roland, CXXXIII) ${ }^{4}$

[Roland A MIS l'olifant à sa bouche, il l'EMBOUCHE fermement, et le JOUE avec grande force.]

Le cadre que décrit ici le premier énoncé, c'est la situation où l'olifant (une sorte de cor) est en contact avec la bouche de Roland. A l'intérieur de ce cadre, ou à partir de cette situation, prennent place les deux éléments narratifs désignés au présent : Roland l'embouche et en joue.

- D'autre part, on le trouve également dans des subordonnées donnant des informations de second plan, comme la relative dans (3).

Einz i ferrai de Durendal asez,

Ma bone espee QUE AI CEINT AL COSTET. (Ch. de Roland, LXXXIV) ${ }^{5}$

[Mais je frapperai à grand coups de Durandal, ma bonne épée QUE J'AI CEINTE À LA TAILLE.]

Ce nouveau temps verbal présente la particularité d'impliquer deux temporalités, correspondant à deux phases de la situation : l'état résultant et la phase processive proprement $\operatorname{dite}^{6}$. A cette étape, on peut décrire la structure informationnelle du PC comme suit: l'état résultant est mis au

4 Les exemples d'ancien et de moyen français ne sont traduits que quand cela nous a paru nécessaire. - Pour les exemples de français classique et moderne, nous indiquons le nom de l'auteur suivi de la date de l'édition. L'ajout d'un 'F' après la date de l'édition indique que l'exemple a été trouvé grâce à la base de données FRANTEXT. Les exemples non référencés ont été forgés pour les besoins de l'exposé, ou résultent de manipulations d'exemples référencés.

5 Ceinte pourrait également être interprété ici comme attribut de l'objet. Pour d'autres exemples de ce type, voir Buridant (2000, § 301-302).

6 On utilisera désormais le terme de «situation» pour désigner tout type de procès dénoté par une forme verbale, quelle que soit la nature de ce procès (transitionnel ou non transitionnel, statif ou non statif, etc.). 
premier plan, il est la phase la plus « saillante », celle vers laquelle la forme verbale vise à diriger notre attention ; la phase processive est quant à elle en arrière-plan.

La deuxième étape va consister à déplacer cette saillance sur la phase processive. Ce déplacement peut s'expliquer par des emplois métaleptiques (métonymie temporelle) ou des emplois consécutifs à une abduction (d'un état observé, on infère le processus ayant produit cet état) ${ }^{7}$. Le PC représentatif de cette nouvelle fonction sera appelé ici «processif». Il faut bien voir cependant que cette dénomination est quelque peu trompeuse, car le déplacement de la saillance sur la phase processive n'est en réalité que partiel ; la résultativité ne disparaît pas complètement. C'est du moins ce que nous tenterons de montrer. Cet emploi du PC est parfois également qualifié d'«antérieur» (e.g. Martin 1971, Vet 1992) ou de perfectum praesens (Schøsler 2004). Sa structure informationnelle est en quelque sorte inverse de celle du PC résultatif (nous verrons toutefois plus loin que cette description doit être nuancée), la phase processive étant au premier plan. On décrira pour l'instant sa fonction comme le fait de désigner (i) une situation passée qui a par ailleurs des conséquences qui perdurent à $\mathrm{T}_{0}$, ou (ii) une situation passée dont l'évocation présente une certaine pertinence à $\mathrm{T}_{0}$. Cette étape est elle aussi complètement réalisée en ancien français :

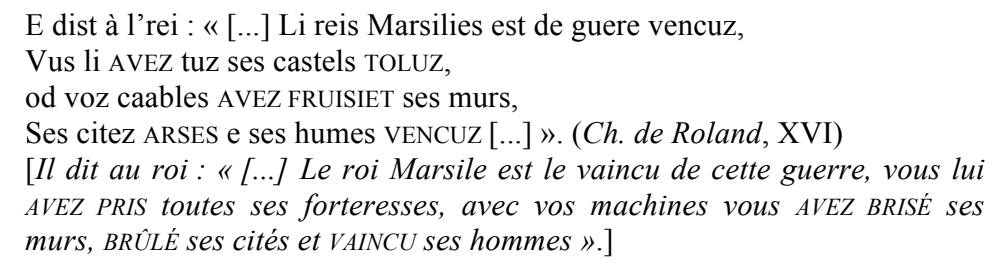

C'est cet emploi que nous analyserons plus en détail dans le présent article. L'un des points qui retiendra notre attention est l'interprétation qu'il convient de donner aux «conséquences » qui perdurent à $\mathrm{T}_{0}$, ou à l'idée de présenter une certaine «pertinence» à $\mathrm{T}_{0}$. Précisons pour l'instant que les $\mathrm{PC}$ correspondant à cette étape ne font pas nécessairement avancer la référence temporelle du texte où ils figurent. Dans l'extrait (4), la séquence formée par avez toluz, avez fruisiet, arses, vencuz (avez pris, avez brisé, brûlé, vaincu) ne vise pas à refléter une séquence chronologique. En ce sens elle ne relève pas de la narration. Tout au contraire il y a, dans cette séquence, suspension de

Ces glissements temporels, souvent assez subtils, peuvent être observés en français moderne. Ainsi, certains adverbes mettent en évidence la phase résultative et, de fait, induisent une lecture résultative; d'autres mettent en évidence la phase processive. Pour une étude de ce phénomène concernant le plus-que-parfait, voir Apothéloz \& Combettes (2011). 
toute chronologie. Chacun des énoncés au PC y est donné comme une " élaboration » de l'énoncé initial Li reis Marsilies est de guere vencuz (Le roi Marsile est vaincu dans la guerre): chacune des quatre situations désignées (avez pris, avez brisé, brûlé, vaincu) indique un ingrédient de la victoire exprimée dans l'énoncé initial. Cette organisation temporelle particulière, ainsi que la relation d'élaboration que ces PC entretiennent avec l'énoncé initial, sont les éléments qui fondent la cohérence de cette séquence, c'est-à-dire en déterminent la logique temporelle aussi bien que les frontières textuelles.

La troisième étape est celle de l'utilisation du PC comme temps de la narration, du moins comme temps ayant perdu tout lien avec le moment de l'énonciation. Nous essaierons plus loin de préciser ce qu'il convient de mettre sous le terme de «narration », qui nous paraît parfois utilisé de manière trop extensive. Il se peut d'ailleurs que cette étape, qui sera appelée simplement « aoriste », ne soit pas encore pleinement réalisée. Quoi qu'il en soit, le critère qui a le plus retenu l'attention des linguistes pour comprendre ce qui se joue dans le passage à cette (éventuelle) troisième étape, c'est la faculté du PC à se combiner avec divers types d'adverbiaux de localisation temporelle. L'intérêt pour ce type d'observation remonte au moins à Maupas (1625). Sur la base de ce critère, il semble que deux périodes doivent être distinguées dans le processus menant à cette troisième étape (cf. Wilmet 1970, Martin 1971, Galet 1974, Caron \& Liu 1999).

Dans la première période, s'étendant de l'ancien français jusqu'au $\mathrm{XVII}^{\mathrm{e}}$ s., le PC n'est pratiquement utilisé que quand la situation est localisée dans un intervalle temporel incluant $\mathrm{T}_{0}{ }^{8}$. Dans les autres cas, c'est presque

Cf. la célèbre préconisation de H. Estienne (1569) connue sous l'appellation de «règle des 24 heures». Cette règle a parfois été interprétée comme si la distinction entre PS et PC reposait sur un critère de distance temporelle. Cependant certains grammairiens du $\mathrm{XVII}^{\mathrm{e}} \mathrm{s}$. avaient bien repéré que la spécificité du PC n'est pas la proximité temporelle, mais le fait que ce temps verbal se combine avec des localisateurs temporels incluant le moment de l'énonciation. De telle sorte que cette semaine appelle un PC, tandis que hier appelle un PS. Dans sa Grammaire, C. Maupas (1625) était tout à fait explicite sur ce point :

Pour mieux entendre cet usage, il faut considerer que les parties du temps sont siècle, an, mois, semaine, jour, ou equivalans. Parlans donc d'une chose advenuë, ou on limite le temps par l'une de ces parties, ou on ne le limite point. Si on ne quote nul terme, vient l'indéfini en usage [le passé indéfini, i.e. le PC]. Exemple. Le Roy a obtenu victoire de ses ennemis, puis leur a pardonné. Mais si l'on assigne quelque certain terme, lors ou il est du tout passé, sans qu'il en reste aucune portion, \& vient en service le Defini [le passé défini, i.e. le PS]. Exemple. L'an mil cinq cens quatre vingts \& dix, le Roy obtint victoire de ses ennemis, 
toujours le PS qui est utilisé. Ainsi, Galet (1974) a montré qu'au XVII s. s., des localisateurs temporels tels que hier, le lendemain ou la veille, qui n'incluent pas $\mathrm{T}_{0}$, sont le plus souvent associés au PS; tandis que des localisateurs comme aujourd'hui ou présentement sont fortement associés au PC. Ces résultats vont dans le même sens que ce que préconisait - ou observait Maupas.

Dans la seconde période, le PC est régulièrement utilisé avec des adverbiaux n'incluant pas $T_{0}$. Cette tendance se manifeste dès la fin du $\mathrm{XVII}^{\mathrm{e}} \mathrm{s}$. et est très affirmée à la fin du $\mathrm{XVIII}^{\mathrm{e}} \mathrm{s}$. Les données obtenues par Liu à partir de corpus épistolaires des $\mathrm{XVII}^{\mathrm{e}}, \mathrm{XVIII}^{\mathrm{e}}$ et $\mathrm{XIX}^{\mathrm{e}}$ s., reprises et analysées dans Caron \& Liu (1999), sont à cet égard extrêmement éloquentes. Pour ne prendre qu'un seul exemple, celui de l'adverbial le lendemain, localisateur temporel anaphorique n'incluant pas $\mathrm{T}_{0}$, les données sont les suivants :

\begin{tabular}{|c|c|c|c|c|c|}
\hline \multicolumn{2}{|c|}{ XVII $^{\mathrm{e}}$ s. } & \multicolumn{2}{c|}{$\mathrm{XVIII}^{\mathrm{e}}$ s. } & \multicolumn{2}{c|}{$\mathrm{XIX}^{\mathrm{e}} \mathrm{s}}$. \\
\hline PS & PC & PS & PC & PS & PC \\
\hline $92.74 \%$ & $7.26 \%$ & $60 \%$ & $40 \%$ & $40 \%$ & $60 \%$ \\
\hline
\end{tabular}

Tableau 1.- Corrélation verbo-adverbiale : pourcentages de PS et de PC avec l'adverbe le lendemain dans un corpus épistolaire

(d'après Caron \& Liu 1999 : 44-45)

Cette tendance est encore plus nette avec d'autres adverbiaux de temps ${ }^{9}$. On a là un indice tout à fait clair de ce qu'on appelle parfois la «dérive aoristique » du PC. Ce phénomène peut également être mis en relation avec la progressive disparition du PS de la langue orale, très avancée à la fin du XVII ${ }^{\mathrm{e}}$ s., selon Fournier (1998).

Cela dit, plusieurs auteurs ont donné des analyses et des arguments solides indiquant que ce processus d'aoristisation était, aujourd'hui, loin

gangna la bataille d'Yvri, peu de temps apres la Ville de Paris se mit en son obeïssance. [...] Ou bien reste encor à passer quelque portion du temps presix \& nommé, lequel est encor en flux, comme parlans du siècle où nous sommes encor, de l'an, du mois, semaine, ou jour qui dure encor, il faut prendre l'indefini. De nostre siecle sont advenuës choses memorables. Il fait beau voir les histoires qu'on a escrites de nostre temps. Cette annee les vignes n'ont point rapporté. Ces deux derniers mois i'ay estudié en la langue Françoise. [...] Parlans des heures ou parties du jour où vous estes encor, vous employerez l'indefini. A matin i'ay esté à l'Eglise, à dix heures i'ay disné, environ midi un mien ami m'est venu voir. (Maupas 1625 : 274-275).

9 Carl Vetters en rappelle ici même quelques résultats. 
d'être complètement achevé (e.g. Revaz 1996, Barbazan 2007, Bres 2010, Rebotier 2014); le PC conserverait donc, quels que soient ses emplois, un lien avec le moment de l'énonciation. Si l'on caractérise la phase aoristique par un basculement complet du côté de la processivité, sans résidu de résultativité, cela signifie que cette troisième phase n'est pas pleinement réalisée concernant le PC. L'un des points que nous aurons à examiner est précisément de déterminer en quoi peut consister ce résidu de résultativité.

$\mathrm{Au}$ fond, l'évolution qu'a subie le PC a donc essentiellement consisté, comme beaucoup de faits diachroniques, en une extension et une diversification fonctionnelles. Elle est à cet égard tout à fait analogue à ce qui a été observé dans l'évolution des parfaits en général (Kuryłowicz 1977, Bybee et al. 1994).

\section{La valeur processive}

Arrêtons-nous maintenant sur la valeur processive du PC et examinons quelles en sont les propriétés et les différentes manifestations en discours.

\subsection{La résultativité et la notion d'énoncé factuel}

Dans le long processus d'extension et de diversification de ses emplois, une propriété au moins du PC demeure constante : c'est le fait d'impliquer deux temporalités. Il s'agit là d'une caractéristique générale des parfaits, comme cela a été observé depuis longtemps (cf. Koschmieder 1929, Comrie 1976) ${ }^{10}$. Elle fait de ces temps verbaux des instruments permettant de mettre en relation deux situations, et explique que dans certaines langues, ils peuvent avoir des usages inférentiels et évidentiels (Dahl 1985, Guentchéva 1990, 1994). Par ailleurs, Weinrich (1964) notait que le PC est un temps qui peine à faire progresser le curseur temporel de la narration (qu'il est, comme on dit aujourd'hui, insuffisamment "propulsif») et nécessite pour cette raison, davantage que le PS, la présence d'adverbes «de consécution narrative». Revaz (1996) note de son côté que même en contexte narratif, le PC présente les situations comme non orientées les unes par rapport aux autres. La bitemporalité du PC est vraisemblablement l'un des facteurs expliquant la relative incapacité de ce temps verbal à fonctionner comme temps propulsif et à orienter les situations les unes par rapport aux autres.

Reprenons la description donnée plus haut du PC processif : désigner une situation passée qui a par ailleurs des conséquences qui perdurent à $T_{0}$, ou dont l'évocation présente une certaine pertinence à $T_{0}$. Cette caractérisation, qu'on rencontre telle quelle ou avec quelques modifications

10 Le processus d'aoristisation, lorsqu'il a atteint son terme, devrait donc aboutir à un temps verbal qui a perdu cette propriété. 
dans la plupart des travaux sur le PC et sur les parfaits, demeure incomplète aussi longtemps qu'on ne précise pas en quoi consistent les «conséquences » en question, ni ce que recouvre, ou peut recouvrir ici, le terme de « pertinence ». En un mot, elle ne dit rien sur les contenus censés être encore d'actualité à $\mathrm{T}_{0}$.

\subsubsection{Contenus de l'état résultant}

Partons du principe selon lequel, au plan aspectuel, le PC oscille entre deux lectures (ou deux emplois): résultative et processive. La première se caractérise par une structure informationnelle dans laquelle la phase résultative, et avec elle un intervalle temporel incluant le moment de l'énonciation, sont mis au premier plan; la seconde se caractérise, symétriquement, par une structure informationnelle dans laquelle la phase processive, et avec elle un intervalle temporel situé dans le passé, sont mis au premier plan. Acceptons également que cette distinction puisse être parfois difficile à mettre en œuvre, notamment quand la forme verbale amalgame les deux lectures. Ce phénomène, noté par Waugh (1987), se manifeste dans l'exemple suivant :

$$
\text { Ce rapport A ÉTÉ MIS EN LIGNE la semaine dernière sur le site de la CRC. }
$$
(Presse, 2013)

D'une part, l'adverbial de localisation temporelle la semaine dernière localise le moment de la mise en ligne du rapport en question et incite par conséquent à analyser ce $\mathrm{PC}$ comme un temps du passé (lecture processive). Mais d'autre part, l'auteur de ce texte veut très vraisemblablement faire savoir également que ce rapport est actuellement en ligne, et donc consultable à $\mathrm{T}_{0}$, au moment où le journal est diffusé (lecture résultative). Il y a donc ici une sorte de continuité entre les deux interprétations du PC, aucune des deux ne l'emportant sur l'autre.

Ceci étant posé, essayons d'examiner quels peuvent être les contenus de l'état résultant dans ces différentes interprétations du PC.

\section{$P C$ résultatif}

S'agissant tout d'abord des emplois résultatifs, ces contenus varient considérablement, selon les propriétés sémantiques du lexème verbal et, plus généralement, de l'expression prédicative. Toutefois, comme l'ont montré Luscher \& Sthioul (1996), deux cas principaux peuvent se présenter :

(i) Celui où le contenu de l'état résultant (que le locuteur veut mettre au premier plan) est déterminé par le sens même du lexème verbal. Cette détermination n'est possible que si le verbe a un sens transitionnel, prédéfinissant un résultat, comme par exemple arriver (interprété comme 
verbe de déplacement) ou s'endormir. Le contenu de l'état résultant peut alors être impliqué par le sens même du verbe : il correspond à quelque chose comme «se trouver à l'endroit vers lequel on se dirigeait» pour arriver, « être endormi » pour s'endormir. C'est ce qui se produit quand les énoncés nous sommes arrivés et il s'est endormi, sont formulés respectivement au moment où se termine un déplacement ou un voyage, et en présence d'une personne qui dort (interprétations résultatives, accomplies).

(ii) Le second cas est celui où le contenu de l'état résultant (que le locuteur veut mettre au premier plan) ne coïncide pas avec un contenu impliqué lexicalement, et doit par conséquent être inféré à partir d'indices contextuels ou situationnels. Contrairement au cas précédent, tout type de verbe peut donner lieu à ce type d'état résultant. Ainsi, nous sommes arrivés (verbe transitionnel) peut être formulé pour faire savoir non pas seulement « nous nous trouvons à l'endroit vers lequel nous nous dirigions », mais aussi par exemple «c'est ici que vous devez descendre »; et j'ai couru (verbe non transitionnel) peut être formulé pour expliquer la raison pour laquelle «je suis essoufflé ».

En d'autres termes, dans les emplois résultatifs, le contenu de l'état résultant peut être déterminé par des éléments aussi bien « sémantiques » (i.e. lexicalement impliqués) que « pragmatiques » (en mettant sous cette étiquette tout ce qui n'est pas lexicalement impliqué). On parlera ci-dessous de "résultativité sémantique » et de « résultativité pragmatique » pour désigner ces deux fonctionnements ${ }^{11}$.

\section{PC processif}

S'agissant des emplois processifs, autrement dit des emplois du PC comme temps du passé, la description du contenu de l'état résultant est plus complexe. Une analyse qui vient immédiatement à l'esprit est celle qui consisterait à considérer que le $\mathrm{PC}$ processif a un fonctionnement inverse du PC résultatif: dans cette perspective, le contenu de l'état résultant du PC processif ne différerait pas de celui du $\mathrm{PC}$ résultatif, mis à part le fait qu'il serait, du point de vue informationnel, en arrière-plan ${ }^{12}$. Cette analyse est toutefois infirmée par un grand nombre de données.

11 Dahl (1985: 134-135) aborde brièvement ce problème en distinguant des états résultants «au sens étroit» et «au sens large». Mittwoch (2008:325) parle quant à elle de résultativité «forte» et de résultativité «faible». Les expressions de "résultativité sémantique » et "résultativité pragmatique » ont été introduites par Apothéloz \& Nowakowska (2010 : 8-10). Sur ce problème, voir également Górnikiewicz (2012).

12 Quoique n'allant pas explicitement jusque-là, certaines affirmations qu'on trouve ici ou là à propos des deux interprétations du PC paraissent bel et bien suggérer ce type d'analyse. Par exemple, à propos de ces deux interprétations, 
En effet, lorsque la résultativité est sémantique, deux cas peuvent se présenter, suivant que l'état résultant a encore cours à $\mathrm{T}_{0}$, ou n'y a plus cours. Cette différence provient du fait que certains verbes transitionnels impliquent des états résultants en principe transitoires (par ex. ouvrir), tandis que d'autres impliquent des états résultants beaucoup plus durables, si ce n'est permanents (par ex. découvrir au sens de «faire une découverte $»)^{13}$. Or, quand l'état résultant impliqué lexicalement est transitoire, il n'est pas possible de le considérer comme valide à $\mathrm{T}_{0}$ si le $\mathrm{PC}$ est employé comme temps du passé. Par exemple, il n'est pas possible de considérer que les états résultants impliqués par les verbes ouvrir et sortir, dans l'extrait ci-dessous, soient encore le cas à $\mathrm{T}_{0}$ :

$$
\begin{aligned}
& \text { En attendant les hors-d'œuvre, elle A OUVERT son sac, en A SORTI un petit } \\
& \text { carré de papier et un crayon [...]. (Camus, L'Étranger, 1942) }
\end{aligned}
$$

En revanche, quand l'état résultant impliqué lexicalement est permanent, il est toujours possible de le considérer comme valide à $\mathrm{T}_{0}$ :

Né le 11 janvier 1906, Hofmann A DÉCOUVERT l'acide lysergique diétylamide, devenu ensuite la drogue préférée du mouvement hippie des années 1960, quand une petite quantité de cette substance a coulé sur sa main lors d'une expérience en laboratoire en 1943. (Presse, 2008)

Dans ce texte, le PC a découvert, compte tenu de la proposition qui lui sert de localisation temporelle (quand une petite quantité de cette substance...), doit indiscutablement être interprété comme référant au passé (il s'agit d'un PC processif). Mais en raison du caractère permanent de l'état résultant impliqué par le verbe découvrir tel qu'il est utilisé ici, il est possible de décrire le résidu de résultativité de cet exemple comme consistant dans la connaissance actuelle que nous avons du LSD, connaissance consécutive à la découverte faite par Hofmann. On voit que, en dépit de temporalités fort différentes, (7) a un fonctionnement analogue à (5).

Cependant, la question demeure de savoir en quoi peut bien consister la résultativité quand l'expression verbale est transitionnelle et implique un état résultant transitoire, comme dans (6). A fortiori cette même question se pose dans le cas des verbes non transitionnels, comme habiter dans (8), puisque ces verbes n'impliquent pas lexicalement un état résultant :

Waugh (1987) parle de focalisation respectivement sur la séquelle (aftermath) et sur l'événement. On rencontre des propos du même type, mais avec un vocabulaire différent, chez Koschmieder (1929) ou chez Martin (1971). L'idée de parler de ces deux phases en termes d'accessibilité, comme le font Caudal \& Vetters (2007), nous paraît procéder de la même logique.

13 Luscher \& Sthioul (1996) parlent respectivement d'implication bornée et d'implication non bornée. 
A cette question, deux types de réponses sont théoriquement possibles : ou bien considérer que dans ces cas, le PC est dépourvu de tout contenu résultatif et ne diffère par conséquent pas du PS ; ou bien maintenir l'idée que ces $\mathrm{PC}$ conservent une certaine résultativité, dont le contenu reste alors à préciser. C'est cette seconde solution qui nous paraît la meilleure, si on veut préserver un minimum de consistance sémantique aux grammèmes de temps verbaux $^{14}$.

\subsubsection{La notion de fait}

La thèse que nous défendrons ici est que la fonction première de tous ces PC processifs n'est pas seulement d'évoquer une situation passée. Elle est aussi de signaler que cette situation a eu lieu. Plus précisément, ces énoncés servent à asserter l'effectivité, au moment de l'énonciation, de l'advenue de la situation qu'ils désignent. Ils indiquent que, à $T_{0}$, l'advenue de la situation est ou doit être considérée comme avérée par le locuteur et son allocutaire. $\mathrm{Si}$ cette description est correcte, elle montre que la «pertinence» pour le moment de l'énonciation, si souvent évoquée à propos du PC processif mais dont le contenu n'est jamais décrit, n'est pas un état résultant au sens ordinaire, purement référentiel, du terme. Certes, dans (7), il s'agit d'informer que la découverte d'un certain acide a été faite par Hofmann ; et, compte tenu du sens du verbe découvrir, il y a indiscutablement ici, comme arrière-plan résultatif, l'information suivant laquelle une certaine connaissance (celle du LSD), auparavant non disponible, est désormais disponible. Mais ce n'est pas cette composante de la résultativité qui nous importe ici. Ce sur quoi nous voulons insister, c'est sur la reconnaissance de l'effectivité de l'événement désigné par la forme verbale. Pour sanctionner terminologiquement cette composante du contenu de l'état résultant, nous dirons que les énoncés comportant un PC processif dénotent des " faits », que ce sont des énoncés «factuels». Cette composante appartient à que nous avons appelé la résultativité « pragmatique ». Arrêtons-nous un instant sur ce terme de « fait ».

Depuis le célèbre article que Vendler (1967) lui a consacré, la notion de fait a donné lieu à une littérature abondante, en particulier dans le champ de la philosophie du langage. Fondamentalement un fait, au sens où nous

14 C'est cette seconde solution qu'adopte Guillaume (1965), mais en s'en tenant à une analyse strictement aspectuo-temporelle. Selon cet auteur, en effet, les verbes n'impliquant lexicalement aucun état résultant sémantiquement consistant, comme marcher, ont aux temps composés un état résultant dont le contenu se limite à de la pure temporalité. 
l'entendons ici, est une situation dont l'advenue est considérée, à un moment repère (dans les exemples ci-dessus, $\mathrm{T}_{0}$ ), comme avérée. Il n'y a donc pas de fait sans sujet de connaissance. Autrement dit, et contrairement à ce que leur appellation pourrait donner à penser, les faits ne relèvent pas de l'« objectivité », du moins si on désigne par ce terme une réalité dont tout sujet connaissant serait évacué. Van de Velde (2006: 85-86) note très opportunément que le concept de «nouveauté », appliqué à un fait, produit une signification dans laquelle la nouveauté est dans la connaissance de la réalité, non dans la réalité elle-même. En d'autres termes, un fait nouveau n'est pas une situation qui vient d'advenir, mais une situation qui vient de parvenir à la connaissance d'un sujet. C'est d'ailleurs ainsi que nous comprenons le mot « fait» dans beaucoup de ses usages, comme par exemple dans l'énoncé : Les juges en charge de l'enquête ont montré qu'ils étaient prêts à étendre leurs instructions en cas de découverte de faits nouveaux (Presse, 2003). Les faits nouveaux susceptibles d'être découverts, ici, sont des situations qui ont déjà eu lieu au moment de l'enquête, mais dont les juges n'ont pas (ou pas encore) connaissance.

Comprise ainsi, la notion de fait suppose donc toujours, outre un repère temporel (i.e. le moment depuis lequel la situation est reconnue comme un fait), un sujet de connaissance, ou de conscience, qui prend en charge le contenu de l'énoncé. Dans la mesure où elle est inséparable de la connaissance, la notion de fait comporte donc une dimension épistémique ${ }^{15}$. Nous tenterons de montrer plus loin (section 4) qu'un énoncé narratif au PS se distingue d'un énoncé factuel précisément par l'absence de cette dimension épistémique et de ce lien à un repère temporel. D'où l'affinité qu'a ce temps verbal avec la fiction.

Dans un exemple comme celui du verbe habiter dans (8), où le verbe n'est pas transitionnel et n'implique donc pas d'état résultant, le seul élément résultatif est précisément cette valeur factuelle. C'est cette valeur qui rattache l'énoncé à $T_{0}$. Nous pensons qu'il en va de même dans (6), où les verbes impliquent lexicalement un état résultant transitoire, qui n'est plus le cas à $\mathrm{T}_{0}$. Ici aussi, seul le composant factuel rattache les énoncés à $\mathrm{T}_{0}$. On peut certes considérer ce court extrait de L'Etranger comme narratif, mais en ce cas il s'agit d'une narration qui se donne des allures de factualité.

\subsection{Variantes de la factualité}

Les énoncés factuels présentent plusieurs variantes. La distinction entre celles-ci n'est pas toujours très tranchée et peut être difficile à établir. On

15 Il nous semble que cette analyse peut être rapprochée de l'affirmation selon laquelle le PC est fondamentalement le temps du témoignage (Barceló \& Bres $2006: 154)$. 
distinguera ici une variante " épisodique », une variante " résomptive » et une variante « expérientielle».

\subsubsection{Variante épisodique et variante résomptive}

La distinction entre énoncé factuel épisodique et énoncé factuel résomptif se justifie pour la raison suivante. Dans des exemples comme ceux examinés plus haut, c'est une situation singulière qui est désignée par les formes verbales. Cette situation peut bien évidemment avoir une durée variable ( $a$ découvert... implique une durée qui n'est pas la même que a habité...), mais elle n'en est pas moins singulière et sa localisation temporelle est définie. Ce type de situation est parfois qualifié d' épisodique» en sémantique temporelle (cf. par exemple de Swart 2010), notamment pour le distinguer des situations « habituelles ». Mais il existe des cas où la factualité concerne une classe de situations et comporte pour cette raison une dimension résomptive. C'est ce qu'on observe dans des exemples comme les suivants :

(9) J'aime mon corps ; IL M'A BIEN SERVI, et de toutes les façons, et je ne lui marchande pas les soins nécessaires. (Yourcenar, Mémoires d'Hadrien, 1951)

[...] adolescent, LA CHASSE AU SANGLIER M'A OFFERT MES PREMIÈRES CHANCES DE RENCONTRE AVEC LE COMMANDEMENT ET LE DANGER ; je m'y livrais avec fureur ; [...] Peut-être N’AI-JE ÉTÉ SI ÉCONOME DE SANG HUMAIN que parce que J'AI TANT VERSÉ CELUI DES BÊTES FAUVES. (ibid.)

Il s'agit dans ces formulations de dire l'effectivité actuelle d'une certaine réalité passée. Mais cette réalité ne relève pas du même niveau que ce qu'on appelle habituellement « événement » ou « procès ». Les situations désignées dans ces exemples sont intrinsèquement collectives (au sens où on dit de certains noms qu'ils sont collectifs), car chacune d'elles inclut une multiplicité indéterminée de situations singulières.

Les observations faites par Fryd (1998: 31-32) sur l'affinité que manifeste le present perfect anglais avec la désignation de «classes d'occurrences de situations ", nous paraissent pointer le même phénomène de résomption, quoique concernant une langue dont le système verbal est différent de celui du français.

Il est intéressant d'observer que cette dimension résomptive peut être associée, par une sorte de figure temporelle, à une transposition dans le futur. Le résultat de cette manœuvre est connu sous l'appellation de futur antérieur (FA) « de bilan ». Ainsi, au lieu de (9), Marguerite Yourcenar aurait pu écrire (plus exactement, aurait pu faire écrire par l'empereur Hadrien), après l'évocation de diverses activités impliquant le corps, le texte (11); et, au lieu de (10), elle aurait pu faire écrire par Hadrien, après l'évocation de ses premières expériences de chasse, le texte (12) : 

marchande pas les soins nécessaires.

(12) [...] la chasse au sanglier m'AURA OFFERT mes premières chances de rencontre avec le commandement et le danger.

Notre analyse du FA de bilan consiste donc à dire que dans cet emploi, le FA projette dans l'avenir (plus exactement, diffère) le statut de fait accordé à la classe de situations désignée ${ }^{16}$. On pourrait ainsi gloser (11) par une formulation comme : «Dans un avenir plus ou moins proche, il apparaitra avéré que, tout bien considéré, mon corps m'a bien servi». Certes, la formulation au FA n'a pas le même sens que celle au PC : le bilan exprimé par le PC ne prend en considération que le passé ; tandis que, en différant ce bilan, le FA laisse ouverte l'éventualité d'autres événements consistant en « mon corps me servir ». Néanmoins, cet emploi du FA peut être considéré selon nous comme une grammaticalisation partielle de la valeur factuelle résomptive.

\subsubsection{Variante expérientielle}

Certains énoncés factuels ont pour fonction, non pas exactement d'asserter l'effectivité de l'advenue d'une situation, mais d'asserter qu'il est effectif qu'un certain type de situation est advenu une fois au moins ${ }^{17}$. Ces énoncés comportent donc un composant existentiel. Soit l'exemple suivant :

Le brochet est le véritable tyran des eaux ; [...] ON L'A VU AVALER DES POISSONS PRESQUE AUSSI GRANDS QUE LUI. (Blanchard, Abrégé d'histoire naturelle, 1809)

Dans cet énoncé, il ne s'agit d'exprimer ni une factualité épisodique, ni une factualité résomptive. La signification de (13) pourrait être glosée par (13') :

(13’) «Il est arrivé (une fois au moins) qu'on le voie avaler des poissons presque aussi grands que lui ».

Autrement dit il s'agit, dans (13), d'asserter qu'un certain type de situation est advenu (a existé) dans le passé, sans préjuger du nombre de fois de cette advenue. La formulation (13) est donc potentiellement itérative. Techniquement, on peut décrire cette signification comme résultant de

16 Sur le rapport entre le FA de bilan, la factualité et les parfaits d'expérience, voir Apothéloz (à paraître).

17 Voici la définition que donne Mittwoch (2008: 324): There is at least one event of the type denoted by the base sentence in the interval terminating at the evaluation time. 
l'application d'un quantificateur existentiel à une situation type, elle-même glosable comme :

(13') « on le voir avaler des poissons presque aussi grands que lui »

Dans la glose (13'), l'expression il est arrivé (une fois au moins) est l'exposant de ce quantificateur. Les situations appartenant à un type intrinsèquement non répétable sont donc, en principe du moins, exclues de cet emploi.

Le quantificateur existentiel, responsable du composant de sens «est arrivé une fois au moins ", implique lui-même un intervalle temporel. En effet, asserter l'advenue d'un type de situation n'est possible que si la validité de l'assertion est envisagée à l'intérieur d'un intervalle déterminé. La plupart du temps cet intervalle demeure implicite, comme dans (13); il est alors construit interprétativement. Dans (13), il pourrait correspondre à l'intervalle qu'indiquerait un complément adverbial comme depuis qu'il fait l'objet d'observations. Il arrive néanmoins que cet intervalle soit formulé. Des expressions comme au cours de $\{\mathrm{ma} / \mathrm{ta} / \mathrm{sa} \ldots\}$ vie ont fréquemment pour fonction d'expliciter ce type d'intervalle. On le désignera par l'expression d'《 intervalle de validation ». S'agissant du PC, il s'étend, par défaut, jusqu'à $\mathrm{T}_{0}{ }^{18}$.

Cet emploi de la valeur de parfait a été décrit depuis longtemps, mais ces descriptions concernent le present perfect anglais. Il est connu sous l'appellation de parfait « existentiel» (McCawley 1971), ou de parfait «d'expérience » ou « expérientiel» (Comrie 1976). La première appellation s'explique par le quantificateur qui entre dans sa description sémantique; la seconde, par l'effet de sens que produit l'énoncé : exprimer l'expérience acquise par un sujet. Dans son ouvrage sur le verbe anglais, Leech $(1971$ : $\S$ $56,57,64)$ en a donné une description minutieuse sous une autre appellation, celle de «passé indéfini » (indefinite past).

Concernant le PC français, mis à part quelques exceptions (e.g. Franckel 1989, Vet 1992, Desclés \& Guentchéva 2003, Karolak 2007, 2008), cet emploi n'est pratiquement jamais mentionné. De façon générale, il semble qu'il ait très peu attiré l'attention des linguistes travaillant sur cette langue. On en trouve pourtant d'assez nombreux exemples dans certains travaux, mais ces exemples sont alors traités soit comme des PC résultatifs, soit

18 De là une condition qui est parfois donnée comme définitoire de ce type d'énoncé : à savoir que la situation désignée doit pouvoir encore advenir à $\mathrm{T}_{0}$, et donc doit être intrinsèquement répétable (Mittwoch 2008). Ce principe de répétabilité a donné lieu à diverses discussions et controverses. Concernant le present perfect anglais, Fryd (1998) et Michaelis (1998) ont montré qu'il n'est pas applicable tel quel. Il en va de même pour le PC français, ainsi qu'un relecteur nous l'a fait remarquer. 
comme des PC processifs. Ainsi Galet (1974: 33), dans son étude des corrélations verbo-adverbiales au $\mathrm{XVII}^{\mathrm{e}}$ s., donne plusieurs exemples où le PC est accompagné de l'adverbial autrefois. Or, certains de ces exemples sont des PC expérientiels: e.g. Rome autrefois a vu de ces émotions (Corneille, Nicomède). De même Wilmet (1997: § 460) analyse le célèbre vers de Nerval : Et j'ai deux fois vainqueur traversé l'Achéron, comme un cas où il y a équilibre entre la valeur d'antériorité et la valeur de présent, autrement dit entre la phase processive et la phase résultative. Or ce PC est un exemple prototypique de PC d'expérience.

Certains auteurs, comme Waugh (1987) ou Vet (1992), considèrent le PC d'expérience comme un cas particulier du PC résultatif. Au plan aspectuel, il nous paraît plus correct de la classer dans la catégorie des processifs. En raison du prédicat d'existence, la phase processive y est en effet davantage focalisée que dans les PC résultatifs.

Il est nécessaire ici de faire une remarque terminologique. La valeur glosée en (13') n'est pas localisée dans la forme verbale seulement (comme pourrait le laisser croire l'appellation de «parfait d'expérience »). Elle résulte en réalité de l'interaction entre le grammème de PC et divers facteurs contextuels. Ainsi, il suffirait de modifier légèrement le texte de (13) pour déclencher une interprétation épisodique, comme le montre (14).

$$
\text { Ce brochet est un véritable tyran des eaux; HIER, ON L'A VU AVALER UN }
$$
POISSON PRESQUE AUSSI GRAND QUE LUI.

Dans ce nouveau contexte, le PC ne désigne pas, comme dans (13), une classe potentielle de situations, mais une situation singulière localisée dans le temps. Il est donc épisodique.

Pour cette raison, et de même que nous avons utilisé plus haut l'expression d' « énoncé factuel », il faudrait parler d' « énoncé d'expérience » plutôt que de « PC d'expérience ». Par commodité nous utiliserons cependant de temps à autres cette dernière expression, mais il est clair que la valeur d'expérience est une propriété d'énoncé et non une propriété de temps verbal. Il en va de même des autres valeurs discutées plus haut.

Ici encore, l'intérêt de distinguer cet emploi des autres emplois du PC trouve sa justification, et toute sa pertinence, dans le fait que la valeur expérientielle a, dans une partie du territoire francophone, une forme grammaticale qui lui est propre : le passé surcomposé (Apothéloz 2010a) (désormais PSC). Il en résulte que dans la partie méridionale de la France ainsi qu'en Suisse romande (domaines d'oc et franco-provençal), le PC de (13) pourrait être remplacé par un PSC :

(15) Le brochet est le véritable tyran des eaux; [...] on l'A EU vU avaler des poissons presque aussi grands que lui. 
On peut donc dire que la signification expérientielle est, dans cette variante de français, grammaticalisée. Cet emploi du PSC est habituellement appelé « régional», mais il serait plus juste de le qualifier d' « existentiel» ou d'« expérientiel ». Cornu (1953) a montré qu'il remonte au moins au XIV ${ }^{\mathrm{e}} \mathrm{s}$. En voici un exemple - nullement exceptionnel - trouvé dans les Registres $d u$ Consistoire de Genève, dans un compte rendu de séance datant de 1547 :

Claude, nepveur de la Charveste.

Auquel fut interroguer s'il sçayt riens de ce que il est le bruyt que la femme de Jehan Roman se gouverne mal. Le nye et en riens savoir. Confesse bien qu'il leur A EU BALLÉ du vin et que... (RCG, 20 janvier 1547)

[Auquel il fut demandé s'il sait que la rumeur court que la femme de Jehan Roman se comporte mal. Il nie en rien savoir. Il confesse quand même qu'il leur A EU LIVRÉ du vin et que...]

Comme nous l'avons suggéré plus haut, certaines données contextuelles sont susceptibles, sinon de déclencher, du moins de favoriser cette signification ${ }^{19}$. Par exemple, dans l'extrait d'audience ci-dessus, le tribunal s'intéresse aux faits et gestes d'un prévenu soupçonné d'avoir livré du vin, et lorsque ce dernier reconnaît l'avoir fait, ce qui importe pour le tribunal, ce n'est pas telle ou telle livraison, mais le fait qu'une telle livraison ait eu lieu (une fois au moins). Ici, le contexte même de l'échange favorise l'apparition de la valeur expérientielle (sans d'ailleurs exclure une autre interprétation). Etant donné que le greffier «possède » un temps grammatical spécialisé pour exprimer cette valeur (le PSC), il l'utilise, rendant ainsi toute méprise impossible.

Cependant, certaines expressions ou formulations sont également associées plus ou moins étroitement à l'interprétation expérientielle. Les principales sont les suivantes : (i) les adverbiaux quantifieurs de fréquence et d'occurrences, (ii) les expressions indiquant l'intervalle de validation, (iii) le déterminant indéfini, (iv) les verbes du témoignage, (v) la mise en série énumérative d'énoncés factuels, (vi) le caractère exceptionnel de la situation, (vii) l'existence d'énoncés plus ou moins figés dans la lecture expérientielle. Il va de soi que ces facteurs sont combinables.

(i) ADVERBIAUX QUANTIFIEURS DE FRÉQUENCE ET D'OCCURRENCES. - Il s'agit, d'une part, d'adverbiaux comme parfois, souvent, rarement, toujours jamais (quantifieurs de fréquence); d'autre part, d'adverbiaux comme plusieurs fois, maintes fois, quelques fois, plus d'une fois, trois fois, etc. (quantifieurs d'occurrences). A ces adverbiaux, il convient d'ajouter déjà interprété comme marqueur existentiel signifiant « une fois au moins »- et

19 On trouve mention de certains de ces éléments dans diverses publications, en particulier dans Leech (1971), Dahl (1985), Guentchéva (1990), Karolak (2007), Mittwoch (2008), Apothéloz (2012). 
qui doit être distingué de déjà marqueur de survenance précoce ${ }^{20}$. Reprenons l'exemple (14) : alors que la formulation on l'a vu avaler un poisson presque aussi grand que lui peut être interprétée aussi bien comme un énoncé factuel épisodique, désignant une situation singulière, que comme un énoncé factuel expérientiel, le seul fait d'insérer l'un des quantifieurs mentionnés ci-dessus exclut l'interprétation épisodique au profit de l'interprétation expérientielle (cf. on l'a \{parfois / déjà, etc.\} vu avaler un poisson presque aussi grand que lui). Voici d'autres exemples où la lecture expérientielle est associée de façon tout à fait caractéristique à un adverbial de ce type :

(17) a. J'ai PARFOIS souri à des gens que je ne trouvais pas du tout drôles. (Gide, Les nouvelles nourritures, $1935, \mathrm{~F}$ )

b. On a SOUVENT comparé le crime à une maladie sociale (Gurvitch, Traité de sociologie, 1968, F)

c. Je l'ai RAREMENT vu travailler avec autant d'ardeur. (Tournier, Vendredi, 1967, F)

d. Vous m'avez montré PLUS D'UNE FOIS que vous vous souciez peu de mon opinion. (Arland, L'Ordre, 1929, F)

Ces adverbiaux ne font en réalité rien d'autre que de spécifier le composant « une fois au moins » de l'énoncé d'expérience.

(ii) EXPRESSIONS INDIQUANT L'INTERVALLE DE VALIDATION. - Il s'agit d'expressions comme dans le passé, dans $\{\mathrm{ma} / \mathrm{ta} / . .$.$\} vie, etc. Les syntagmes$ prépositionnels ou les subordonnées en depuis peuvent également désigner cet intervalle. Ainsi (18) serait, contrairement à (14), préférentiellement interprété comme un énoncé d'expérience.

DEPUIS QUE JE L'OBSERVE, je l'ai vu avaler un poisson presque aussi grand que lui.

(iii) DÉTERMINANT INDÉFINI. - On observe également une certaine affinité entre l'interprétation expérientielle et le déterminant indéfini. Plus exactement, la détermination nominale indéfinie est susceptible de déclencher une interprétation expérientielle du temps composé, notamment quand elle concerne le second actant (Karolak, 2008) et qu'elle est au pluriel. On le voit en comparant les formulations suivantes :

20 Déjà est un marqueur existentiel dans Avez-vous déjà mangé de la viande avariée? (pour signifier, par exemple, "savez-vous quel goût a la viande avariée ?»). Il est en revanche un marqueur de survenance précoce dans $I l$ est déjà onze heures. 
(19) a. Elle a traduit CE roman.

b. As-tu mangé LE bortsch ?

c. J'ai vu LE noyé.
Elle a traduit DES romans.

As-tu mangé DU bortsch?

J'ai vu UN noyé.

Les formulations de la colonne de droite admettent une lecture expérientielle, même si ce n'est pas la seule lecture possible. Pour favoriser encore davantage cette lecture, il suffirait d'ajouter l'adverbe déjà, qui serait alors interprété comme signifiant « une fois au moins » (cf. J'ai déjà vu un noyé). En revanche, aucune des formulations de la colonne de gauche n'admet cette lecture; toutes désignent une situation épisodique. Déjà ajouté à ces formulations produirait la signification de survenance précoce ou une autre signification encore, mais certainement pas la signification expérientielle.

(iv) VERBES DU «TÉMOIGNAGE ». - On note également une affinité toute particulière des énoncés d'expérience avec les verbes signifiant le témoignage, spécialement quand ces verbes sont conjugués à la $1^{\text {ère }}$ personne. Il s'agit principalement des verbes voir, entendre, connaître - cf. exemple (19c) ci-dessus ainsi que (20) ci-dessous.

(v) ÉNUMÉRATION. - Il a également été noté que les énoncés d'expérience ont une certaine prédilection pour les énumérations. Il y a alors suspension de la chronologie, et toute la séquence est au service d'une même idée.

J'AI VU beaucoup d'individus, j'AI VISITÉ quelques nations, j'AI PRIS ma part d'entreprises diverses sans les aimer, j'AI MANGÉ presque tous les jours, j'AI TOUCHÉ à des femmes. (Valéry, La soirée avec M. Teste, 1896, cité par Weinrich $1973: 92$ )

(vi) CARACTÈRE EXCEPTIONNEL DE LA SITUATION. - C'est ici un facteur qui n'a rien de formel ou de grammatical, et qui relève des représentations et savoirs sur le monde, de ce que Grize (1996) appelait les «préconstruits culturels ». On observe en effet que, plus la situation désignée apparaît comme improbable, inattendue, etc., plus l'interprétation expérientielle est favorisée. On le voit tout particulièrement dans les questions. Les exemples suivants ont été mis dans un ordre reflétant une sorte de gradient de probabilité (du plus au moins probable). Toutes choses égales par ailleurs, on est davantage enclin à interpréter comme expérientiel le dernier exemple que le premier $^{21}$.

21 Cette corrélation entre improbabilité et interprétation expérientielle est à l'origine d'une erreur de diagnostic assez fréquente concernant le PSC dans sa variante régionale. Certains linguistes ont en effet affirmé que la signification de cette forme comportait une indication de rareté ou d'exceptionnalité. Or, ce n'est pas le grammème de PSC en lui-même qui comporte cette indication. $\mathrm{Si}$ elle existe, elle est seulement due à la valeur d'expérience que code le PSC régional, et au fait que cette valeur a de fortes affinités avec des faits jugés 
(21) Avez-vous mangé de la viande ?

Avez-vous mangé de la viande de cheval?

Avez-vous mangé de la viande de chameau?

Avez-vous mangé des insectes?

(vii) ÉNONCÉS EXPÉRIENTIELS FIGÉS. - Enfin, notons que certaines formulations brèves, plus ou moins figées, ne sont pratiquement utilisées que pour produire des énoncés expérientiels. Certaines d'entre elles sont étroitement associées aux formes comparatives, et on y retrouve les verbes du témoignage : j'ai vu mieux - on a vu pire - j'ai entendu pire - il a fait mieux - j'ai connu plus moche, etc. Ici encore, la seule présence d'une expression de localisation temporelle suffit à défiger l'énoncé et à le transformer en énoncé factuel épisodique (cf. La semaine dernière, j'ai entendu pire).

Il est important de rappeler que cette valeur d'expérience est associée aux parfaits en général. En conséquence elle concerne également, pour ce qui est du français, le plus-que-parfait (PQP) et le FA. En ce cas, l'intervalle de validation a une borne terminale qui se situe respectivement dans le passé (pour le PQP) et dans le futur (pour le FA). Il en va de même de la localisation temporelle du repère à partir duquel la situation désignée est considérée comme avérée. C'est ce qu'on observe dans les deux exemples suivants :

(22) Celui qui l'inquiétait le plus était le président [...]. IL L'AVAIT PARFOIS CROISÉ DANS LA DEMEURE FAMILIALE BRESTOISE... (Keriel, Les Serres d'Orchidée, 2006)

Avant qu'un nouveau droit soit sorti de ce chaos, LES ASTRES SE SERONT SOUVENT LEVÉS ET COUCHÉS. (Chateaubriand, Mémoires d'outre-tombe, $1849, \mathrm{~F})$

$\mathrm{Au} F A$, il existe également quelques tours plus ou moins figés dans la valeur d'expérience, comme : on aura tout vu, on aura tout entendu.

Pour conclure sur cette question, on notera que les énoncés d'expérience, quoique référant indiscutablement au passé (quand ils sont au $\mathrm{PC}$ ou au PQP), sont étrangers au genre narratif. Ils peuvent certes intervenir dans des séquences narratives, mais ils forment alors des îlots de factualité. Leur environnement discursif le plus naturel est, tout au contraire, le genre argumentatif, comme nous avons essayé de le montrer ailleurs (Apothéloz 2010b). Si l'on définit un argument comme consistant dans l'articulation d'une «thèse » (pouvant d'ailleurs être implicite) et d'un certain nombre d'énoncés justifiant ou étayant cette thèse, on constate alors que les énoncés

inhabituels ou inattendus. On la rencontre aussi bien avec le PC quand il est utilisé dans sa valeur d'expérience. 
d'expérience assument la plupart du temps cette fonction de justification ou d'étayage.

\section{Précisions (et malentendus) sur la narrativité}

Dans les discussions sur la valeur d'aoriste et sur l'aoristisation du PC, la narration comme genre occupe en général une place importante. Le célèbre article où Benveniste (1959) oppose énonciation de discours et énonciation historique y est évidemment pour quelque chose. Nombre d'auteurs considèrent en effet que le $\mathrm{PC}$ ne peut être interprété comme un aoriste que quand il est utilisé comme temps principal de la narration. Mais qu'est-ce au juste qu'une narration? A voir la façon dont ce terme est parfois utilisé pour commenter certains exemples, il n'est peut-être pas inutile de reposer ici cette question. Prenons deux textes à titre d'exemple. Le premier est un extrait d'une lettre de Pascal, cité et commenté par Caron \& Liu (1999 : 38) :

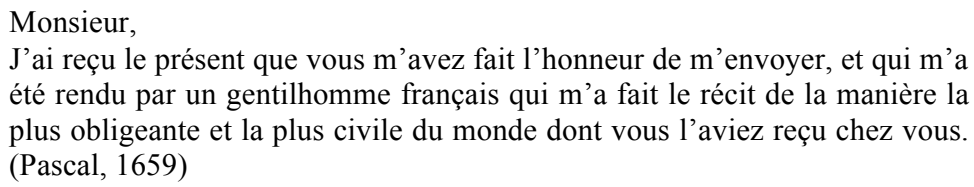
été rendu par un gentilhomme français qui m’a fait le récit de la manière la plus obligeante et la plus civile du monde dont vous l'aviez reçu chez vous. (Pascal, 1659)

Les auteurs donnent cet extrait pour accréditer l'idée qu'au XVII ${ }^{\mathrm{e}}$ s., le temps le plus utilisé lorsqu'il s'agit de faire de la narration dans le passé, du moins dans la correspondance, est le PC. Pourtant, on peut légitimement se demander si ce texte relève bien de la narration. Cette question revient, de fait, à s'interroger sur ce qui différencie le PC processif factuel et un PC qui serait véritablement aoristique (si ce dernier existe).

L'une des caractéristiques principales de la narration est la successivité temporelle des situations désignées. Plus précisément il faut, pour qu'il y ait narration, que l'ordre des énoncés soit un reflet au moins partiel de l'ordre chronologique supposé des situations désignées. Pour rendre compte de ce parallélisme, on dit souvent qu'il y a, dans une narration, un rapport d'iconicité entre l'ordre dans lequel les situations sont évoquées et l'ordre dans lequel elles ont supposément eu lieu. Or tel n'est pas le cas dans cette lettre de Pascal. Pour le faire voir, posons les définitions suivantes, qui ne font que reprendre, dans l'ordre où elles sont désignées, les cinq situations qui composent ce texte.

$\mathrm{S} 1=$ j'ai reçu le présent

$\mathrm{S} 2$ = que vous m'avez fait l'honneur de m'envoyer

$\mathrm{S} 3$ = qui m'a été rendu par un gentilhomme français

$\mathrm{S} 4$ = qui m'a fait le récit

$\mathrm{S} 5=$ de la manière $[. .$.$] dont vous l'aviez reçu chez vous$ 
L'ordre chronologique de ces cinq situations est vraisemblablement le suivant (à gauche, la situation la plus ancienne, à droite le moment de l'énonciation $\mathrm{T}_{0}$ ) :

$$
\mathrm{S} 5<\mathrm{S} 2<\{\mathrm{S} 1, \mathrm{~S} 3\}<\mathrm{S} 4<\ldots \mathrm{T}_{0}
$$

Certes, S5 ne peut pas être traitée sur le même plan que les autres situations, étant donné qu'elle est formulée au moyen d'un PQP. Néanmoins, comme on le voit, l'ordre dans lequel les situations sont désignées est passablement différent de celui dans lequel elles ont eu lieu.

Par ailleurs - et bien que ce point soit plus difficilement vérifiable -, il ne fait guère de doute que les situations relatées par ces PC sont toutes susceptibles d'être dotées d'une certaine pertinence à $\mathrm{T}_{0}$, pour l'auteur de la lettre comme pour son destinataire. Pour ces deux protagonistes, elles ont une certaine « actualité ».

Cette analyse indique que nous sommes relativement loin ici du genre narratif. L'auteur de cette lettre ne narre pas, il informe. La pertinence et l'actualité de ces énoncés au PC réside dans leur caractère factuel. Selon la terminologie que nous avons établie, ce sont des énoncés factuels épisodiques. Aspectuellement, ces PC sont processifs, mais ils conservent un résidu de résultativité, qui est précisément leur factualité. Pour cette raison, il paraît difficile de les considérer comme des aoristes. En réalité il s'agit, dans ce texte, non pas d'immerger le destinataire dans le narratif, en lui faisant oublier tout ce qui relève du moi-ici-maintenant, mais de l'informer que certains événements sont advenus.

Second exemple, caractérisé de narratif cette fois-ci par Martin (1971), et qui selon nous ne relève pas non plus de la narration stricto sensu:

(25) Lesquels [= les Français] paravant la foi reçue, et depuis par armes et corporelle valeur, ONT ACQUIS la dernière régnation, et après plusieurs fois avoir concresté en leur terroir, à toutes roides puissances ONT ENVAHI les Italies, et par le destroit des Alpes horribles ONT MESME ESPOUVANTÉ les Romains, SAISI couronne impériale et thrône de souveraine sacrée majesté ; ONT AUSSI PARTRASSÉ toute la Germanie et SOUMISE à leur dition ; ployèrent les Grecs, réduisirent les Espagnes, donnèrent règle aux terres maritimes, et tout l'enclos d'entre les deux mers haut et bas soumirent à leurs lois et obéissance; (Chastellain, 6-7. In Martin 1971: 397, les italiques sont de Martin)

[Lesquels, ayant auparavant reçu la communion, ONT ensuite ACQUIS par les armes et la force physique le dernier royaume, et après avoir plusieurs fois agrandi leur territoire, avec des moyens vigoureux ONT ENVAHI les Italies, [...] ONT MÊME ÉPOUVANTÉ les Romains, SAISI la couronne impériale [...] ; ils ONT AUSSI PARCOURU entièrement la Germanie et l'ONT SOUMISE à leur autorité...] 
Ici, contrairement à l'exemple précédent, les situations désignées par les PC pourraient, à partir de ont envahi les Italies, former une succession temporelle. On peut cependant noter que cet extrait comporte des marqueurs qui, eux, renvoient à tout autre chose que de la pure narration : il s'agit de même et aussi (cf. ont MESME espouvanté les Romains, ont AUSSI partrassé toute la Germanie). Ces adverbes sont typiquement argumentatifs ${ }^{22}$. Ils indiquent que dans cet extrait, une relation argumentative est instaurée entre l'auteur et ses lecteurs. Le lecteur n'est pas, dans ce texte, immergé dans le narratif; la séquence est formulée en fonction d'une certaine finalité, elle vise à étayer pour son lectorat une certaine représentation concernant les Français, elle exprime un point de vue sur leurs faits d'armes ${ }^{23}$. Chaque situation évoquée contribue, à un titre ou à un autre, à cette finalité. On notera d'ailleurs que la dimension argumentative perturbe ici la compréhension de la chronologie des événements. Ainsi, ce qui est dit à propos de la Germanie pourrait s'être passé aussi bien avant qu'après ce qui est dit des Italies et des Romains. Cela est dû aux adverbes aussi et même, qui mettent en quelque sorte les quatre situations désignées (ont envahi, ont épouvanté, ont parcouru, ont soumis) sur un même plan par rapport au but argumentatif visé. Pour toutes ces raisons, on peut considérer que cet extrait ne relève pas de la narration, du moins pas de la narration pure. Son sens est essentiellement factuel et argumentatif.

L'analyse de ces deux exemples montre que l'utilisation du PC comme temps du passé, donc à dominante processive, si elle est bien une condition nécessaire pour qu'on puisse dire qu'il y a narration, n'en est pas pour autant une condition suffisante.

Pour qu'il y ait narration, plusieurs conditions doivent être réunies. Toutes n'ont probablement pas la même importance, mais il est utile d'en dresser la liste. Tout d'abord, il faut que le temps verbal soit processif et borné, et donne de la situation une représentation globale (Bres 2010). Dans la narration pure, il doit même être dépourvu de tout résidu de résultativité. La narration pure suppose en effet une suspension de la relation interlocutive ${ }^{24}$. Le temps verbal doit également être «propulsif» (Johanson

22 Même adverbe est explicitement décrit comme argumentatif par le Dictionnaire du moyen français hébergé sur le site de l'ATILF (www.atilf.fr/dmf). En pragmatique, même est décrit comme un adverbe typiquement argumentatif indiquant que la situation désignée est, davantage qu'une autre situation, susceptible d'étayer une certaine assertion (Ducrot 1983).

23 Que cette représentation ou ce point de vue soient explicitement formulés est un autre problème.

24 Barbazan (2007) a développé une argumentation détaillée pour montrer que le PS est précisément caractérisé par la suspension de tout composant allocutif, contrairement au PC. Sur ce point, on peut également renvoyer à la façon dont Benveniste (1959) définissait l'énonciation « historique » (récit). 
2000), i.e. avoir la propriété de faire «avancer» l'action, de créer des successivités temporelles dans lesquelles chaque situation désignée suppose une situation antérieure et crée l'attente d'une situation ultérieure. Dans les séquences ainsi formées, l'ordre des situations est iconiquement simulé par l'ordre des énoncés désignant ces situations. Cette horloge implicite a pour conséquence que chaque situation de la séquence narrative a une localisation temporelle définie par défaut ${ }^{25}$ (alors que la lecture factuelle s'accommode sans difficulté d'une localisation temporelle indéfinie). Le PS, tel qu'il est utilisé comme temps conducteur de la narration, remplit très exactement toutes les conditions de cette machinerie narrative. Que, suite à un processus d'aoristisation, le PC s'y soit aujourd'hui en partie substitué et ait progressivement conquis certains emplois narratifs n'est pas contestable. En revanche, il est à peu près certain que dans ces emplois, il conserve un lien avec $\mathrm{T}_{0}$. Son aoristisation n'est donc pas complète, et ce lien avec l'énonciation est précisément, selon nous, ce qui constitue sa factualité. Il y aurait donc deux types de narrativités: l'une factuelle ou simulant la factualité, l'autre non factuelle ou simulant la non-factualité.

\section{PC factuel et PS dans un corpus de moyen français}

On situe habituellement la période du moyen français entre le début du $\mathrm{XIV}^{\mathrm{e}}$ s. et la fin du XV $\mathrm{XV}^{\mathrm{e}}$ s. Selon Martin (1971), le système des temps verbaux du moyen français, surtout vers la fin de cette période, diffère assez peu de celui du français moderne. Les principales différences concernent l'usage du PS, du PC et du PQP, qui se répartissent autrement qu'aujourd'hui leurs territoires fonctionnels. Ainsi le PS a, en ancien et moyen français, des emplois beaucoup plus diversifiés qu'en français classique. Il s'agit là d'un héritage du parfait latin (Mellet 2000). On le rencontre notamment :

- Quand il s'agit de désigner une situation antérieure à une autre situation elle-même exprimée par un PS - autrement dit dans des contextes où on attendrait, en français moderne, un PQP. Cette liberté qu'a le PS de l'ancien et du moyen français de quitter la chronologie de la narration pour référer à n'importe quel moment du passé est, du point de vue du français moderne, tout à fait frappante.

(26) L'andemain de la venue du roy, FUT DECAPITÉ monseigneur Pierres de Saquainville qui FUT PRINS à la bataille de Cocherel [...]. (Valois < Martin $1971: 392)$

25 «Par défaut» est important, car on sait que les exemples abondent en français moderne où le PS n'est pas astreint à l'ordre chronologique des situations. 
- Avec des lexèmes verbaux statifs, dans des énoncés dont la fonction textuelle est de donner des informations d'arrière-plan relativement au premier plan de la narration. On aurait ici en français moderne un IMP.

«Elles, qui FURENT honteuses de sa venue, acoururent vers leur damme, qui fut vergongneuse de ce que elle estoit nue [...] (Froissart < Martin 1971 : 176)

Martin explique ces PS statifs et quasi imperfectifs, très fréquents en ancien et moyen français, en faisant l'hypothèse que l'ancienne langue, pour mettre en contraste premier plan narratif et informations de second plan, utilise davantage les ressources de l'aspect lexical que celles de l'aspect grammatical. Il y aurait donc dans ces PS « compensation de l'aspect par la modalité d'action » (Martin 1971 : 175 sqq.) ${ }^{26}$.

Ce phénomène n'a rien d'exceptionnel. On pourrait le comparer, mutatis mutandis, à ce qu'on observe avec le présent de narration. Quand un locuteur produit une séquence narrative au présent, il se prive par là même de la possibilité de distinguer par des moyens grammaticaux (i.e. par le choix des temps verbaux) entre un énoncé désignant une situation du premier plan, et un énoncé désignant une situation de second plan. Mais la distinction de ces deux statuts informationnels n'en est pas pour autant abolie; elle demeure présente, mais comme un fait purement interprétatif. C'est ici que l'aspect lexical (la «modalité d'action » de Martin) joue un rôle important. Ces considérations indiquent que ce phénomène de compensation doit être considéré comme relevant moins du système de la langue, que de la façon dont ses usagers la mettent en œuvre dans le cadre de certaines conventions ou pratiques rédactionnelles.

- Dans l'ancienne langue, on rencontre également le PS dans des subordonnées en depuis :

(28) [...] depuis le temps que je vEINS en son service, jusques à l'heure de son trespas, où j'estoye present, ay faict plus continuelle residence avec luy que nul autre [...] (Comm., Prologue)

[depuis l'époque où j'ENTRAI à son service jusqu'à l'heure de sa mort, où j'étais présent, j'ai plus vécu en sa compagnie que nulle autre personne.]

- On le rencontre également dans des complétives dépendant d'une proposition principale dont le verbe est au PR ou au PC :

(29) Il m'a autresfois dit qu'il trouva une nuyct la bastille Sainct Anthoine ouverte par la porte des champs, de nuyct [...]. (Comm. I, 11)

26 Voir Schøsler (1994) pour une discussion critique de cette explication. 
- Compte tenu de ce que nous avons exposé à propos des énoncés d'expérience, on peut maintenant ajouter à cette liste, précisément, les énoncés d'expérience. A notre connaissance, les travaux sur le moyen français n'identifient jamais cet emploi comme relevant d'un type spécifique.

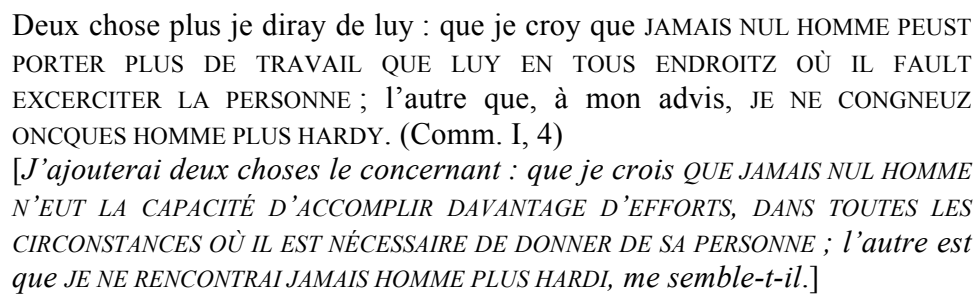
N'EUT LA CAPACITÉ D'ACCOMPLIR DAVANTAGE D'EFFORTS, DANS TOUTES LES CIRCONSTANCES OUं IL EST NÉCESSAIRE DE DONNER DE SA PERSONNE ; l'autre est que JE NE RENCONTRAI JAMAIS HOMME PLUS HARDI, me semble-t-il.]

$\mathrm{Au}$ total, on constate qu'en moyen français, le PS est un temps passablement sous-déterminé au plan aspectuel. Il est beaucoup moins exclusivement associé à la narrativité, au sens étroit du terme, qu'il ne le sera à partir du français classique. De plus, il ne manifeste aucune incompatibilité avec les énoncés d'expérience.

Nous allons maintenant examiner systématiquement tous les PC processifs dans les Livres I à III des Mémoires de Philippe de Commynes $(1447-1511)^{27}$. Cette partie des Mémoires a été rédigée entre 1489 et 1491, c'est-à-dire dans la dernière période du moyen français. Elle constitue un corpus de 64200 mots, ce qui représente un peu plus de 3 fois le volume du présent article. Nous laisserons complètement de côté ici les PC résultatifs, qui ne nous concernent pas directement dans ce travail. Rappelons que notre objectif est d'étudier le rôle de la factualité, et notamment des énoncés d'expérience, dans l'extension du PC et l'évolution des rapports PS/PC.

\section{1. Énoncés factuels épisodiques et résomptifs}

Tous les PC processifs rencontrés dans le texte de Commynes comportent un élément résultatif et sont donc factuels au sens où ce terme a été défini. Par ailleurs, nous n'avons trouvé aucun exemple de séquence de PC qui pourrait être décrite comme une séquence narrative. En fait, quand il n'est pas lié à la valeur expérientielle, le PC processif intervient dans des environnements extrêmement typés. Pour l'essentiel ces environnements sont les suivants :

(i) En premier lieu, on le rencontre de façon récurrente dans des propositions à caractère méta-textuel permettant de repérer, dans la chronologie du texte, un moment antérieur ou postérieur à celui où en est arrivé le texte. On voit alors systématiquement apparaître des verbes comme dire ou parler à la $1^{\text {ère }}$ pers. (désignation de l'auteur), oü̈r, entendre ou voir à

27 L'édition utilisée est indiquée dans la bibliographie finale. 
la $2^{\mathrm{e}}$ pers. (désignation du lecteur), avec parfois la référence à un «lieu » du texte (ailleurs, ci-dessus, au commencement de ce propos), voire au texte proprement dit (ce propos, notre propos, ces mémoires). En un mot, l'usage du PC est ici associé aux différentes manifestations de la déixis textuelle. De façon générale, on observe dans ces propositions une forte prédominance de la $1^{\text {ère }}$ personne.

Sur le plan de la syntaxe, ces propositions sont souvent de «second rang ». Nous rangeons sous cette appellation les propositions subordonnées servant à apporter des informations annexes, d'importance secondaire, comme dans les exemples ci-dessous. Il y a alors, au moment où intervient cette proposition, décrochage du cours de la narration. Tous ces emplois sont factuels.

(31) Et toute ceste bende DONT J'AY PARLÉ arriva auprès de Peronne, comme le roy entroit. (Comm. II, 6)

(32) Et en parlant, le seigneur de Contay, bien saige homme (COMME JE vOUS AY DIT AILLEURS), luy dist que [...]. (Comm. I, 12)

(33) $[\ldots]$ et grant nombre de chevaliers et escuyers, dont les aucuns avoyent esté en Bourbonnoys, COMME J'AY DIT AU COMMENCEMENT DE CE PROPOZ. (Comm. I, 6)

(34) Après, en demanda au seigneur de Contay, QUE PLUSIEURS FOIS AY NOMMÉ [...]. (Comm. II, 1)

(35) [...] et y en avoit six de ce nombre des ostaiges, que peu de jours avant avoit delivréz, avecques les condicions QUE AVEZ ENTENDUES CY-DESSUS. (Comm. II, 2)

Lorsque la situation désignée est explicitement localisée dans le passé (e.g. que avez entendues CY-DESSUS), la phase processive est clairement mise en saillance, mais la phase résultative n'en demeure pas moins bien présente.

Il est fréquent également de trouver un PC factuel dans un énoncé, voire dans une séquence, entièrement dévolus à cette fonction méta-textuelle. Ces séquences servent alors à justifier un développement ou une digression (en ce sens elles sont argumentatives), à faire un rappel ou une annonce, à récapituler une séquence narrative - bref à baliser le texte et à en guider la lecture.

(36) Retournant à nostre propoz, vOUS AVEZ OUY COMME, au partir de Louvain, LEDIT DUC MIST LE SIĖGE DEVANT SAINCT TROND ET ASSORTIT SON ARTILLERIE. (Comm. II, 2)

(37) J'AY BEAUCOUP MIS AVANT RETOURNER À MON PROPOZ DE L'ARREST en quoy estimoit le roy estre à Peronne, DONT J'AY PARLÉ PAR CY-DEVANT, et EN SUYS SAILLY pour dire mon advis aux princes de telz assemblées. (Comm. II, 9) [J'AI CONSACRÉ BEAUCOUP DE TEMPS, AVANT DE REVENIR À MON PROPOS, À L'ÉTAT DE CAPTIVITÉ dans lequel le roi estimait être à Peronne, DONT J'AI 
PARLÉ CI-DESSUS, et J'AI LAISSÉ CE SUJET pour donner mon opinion aux princes à propos de telles réunions.]

Le point commun à tous ses emplois est que le $\mathrm{PC}$ intervient ici dans ce que Vuillaume (1990) appelle la « fiction secondaire». Le processus de la lecture est pris en compte et constitue une temporalité en soi, habitée par les événements que sont les énonciations successives produites par le narrateur. Dans cette fiction secondaire, le narrateur « se donne les allures d'un conteur qui s'adresse oralement à son public » (Vuillaume 1990:30).

(ii) Une deuxième fonction à laquelle les PC factuels non expérientiels sont fréquemment associés est celle du commentaire. Il ne s'agit pas ici du registre du métadiscours, comme dans le cas précédent. L'auteur quitte certes momentanément le cours de la narration, mais pour donner une information complémentaire concernant par exemple le devenir ultérieur de l'un des protagonistes, comme dans (38)-(39), pour formuler un jugement personnel, comme dans (40), indiquer ses sources, ajouter une information présentant une certaine pertinence compte tenu du propos en cours, comme dans (41), anticiper sur l'avenir, comme dans (42). ${ }^{28}$

(38) Et ledict conte fut en très grant dangier et eut plusieurs coups, et entre les autres ung à la gorge d'une espée, DONT L'ENSEIGNE LUY EST DEMOURÉE TOUTE SA VIE [...]. (Comm. I, 4)

[Ledit conte fut en très grand danger et reçut plusieurs coups, dont un à la gorge par une épée, DONT LA CICATRICE LUI EST RESTÉE TOUTE SA VIE.]

(39) $[\ldots]$ et fut mys le roy Henry $\mathrm{VI}^{\mathrm{e}}[\ldots]$ en prison au chasteau de Londres et declairé traistre et crimineulx de lèse majesté, OÙ IL A USÉ LA PLUSPART DE SA VIE ET À LA FIN A ESTÉ TUÉ. (Comm. I, 7)

(40) Des jeunes y avoit-il assez. Entre les autres ung fort bien renommé appellé messire Philippes de Lalain, qui estoit d'une race DONT PEU S'EN EST TROUVÉ QUI N'AYENT ESTÉ VAILLANS ET COURAGEUX, ET PRESQUE TOUS MORTZ EN SERVANT LEURS SEIGNEURS EN GUERRE. (Comm. I, 2)

(41) Je ne luy ouy oncques dire qu'il fust las ny ne luy vey jamais faire semblant d'avoir paour, ET SY AY ESTÉ SEPT ANNÉES DE RENG EN LA GUERRE AVECQUES LUY [...]. (Comm. I, 4)

[Jamais je ne l'entendis dire qu'il était las ni ne le vis laisser paraître qu'il avait peur, ET POURTANT J'AI ÉTÉ SEPT ANS DE SUITE À LA GUERRE AVEC LUI.]

(42) Ces parolles esjouyrent fort ledict duc, et incontinent fut apporté ledict traicté de paix [...]. Et tantost furent sonnées les cloches par la ville, et tout le monde fut fort esjouy. AUtresfois a PLEÜ AU ROY ME FAIRE CEST HONNEUR QUE DE DIRE QUE J'AVOYE BIEN SERVY À CESTE PACIFICATION.

28 Ces informations d'arrière-plan doivent être distinguées de celles qui sont apportées par des PQP à interprétation résultative, comme dans l'exemple suivant: Dès qu'ilz vindrent à la rivière, ilz y feirent mettre ces bateaulx $Q U^{\prime} I L Z$ AVOYENT APPORTEZ [...]. (Comm. I, 6) 
Incontinent escripvit ledict duc ces nouvelles en Bretaigne [...]. (Comm. II, 9).

[Ces paroles réjouirent beaucoup ledit duc, et immédiatement fut apporté ledit traité de paix. Bientôt on sonna les cloches dans la ville, et tout le monde fut fort réjoui. PAR LA SUITE, IL A PLU AU ROI DE ME FAIRE L'HONNEUR DE DIRE QUE J'AVAIS ÉTÉ POUR BEAUCOUP DANS LA CONCLUSION DE CETTE PAIX. Ledit duc écrivit immédiatement ces nouvelles en Bretagne.]

Le changement de registre textuel que produit ce type de PC apparaît clairement dans le dernier exemple. L'abandon de la narration, d'ailleurs de courte durée, y est marqué en effet non seulement par la transition PS $>$ PC, mais également par l'adverbe autresfois (qui signifie ici «à un autre moment », « par la suite »).

Une autre expression est systématiquement associée à ce type de rupture temporelle : il s'agit de depuis adverbe ou préposition ${ }^{29}$ :

(43) Là arrivèrent messire Charles de France, lors duc de Berry, seul frère du roy, le duc de Bretaigne, monsr de Dunoys, monsr de Dampmartin, monsr de Loheac, monsr de Bueil et monsr de Chaulmont et messire Charles d'Amboyse, son filz, QUI DEPUIS A ESTÉ GRAND HOMME EN CE ROYAULME [...]. (Comm. I, 5)

(44) [...] par la main de messire Tannneguy du Chastel, QUI DEPUIS A ESTÉ GOUVERNEUR DE ROUSSILlON ET EN AUCTORITÉ EN CE ROYAUlme. (Comm. I, 1)

(45) Et, sur ceste seulle ymagination, fut envoyé messire Guillaume de Cluny, prothonotaire, QUI EST MORT DEPUIS EVESQUE DE POICTIERS, devers le roy Edouart d'Angleterre, qui pour lors régnoit. (Comm. I, 5)

[Et, sur cette seule idée peu fondée, fut envoyé messire Guillaume de Cluny, protonotaire, QUI PAR LA SUITE EST MORT ÉVEEQUE DE POITIERS, auprès du roi Edouard d'Angleterre, qui régnait alors.]

(46) Ledict duc Philippes, QUI, DEPUIS SA MORT, A ESTÉ APPELLÉ LE BON DUC PhILIPPES, consentit que [...]. (Comm. I, 2)

Toutes ces séquences au PC sont factuelles et réactivent la relation d'interlocution, normalement occultée dans les séquences narratives. Le fait qu'y apparaisse parfois la $1^{\text {ère }}$ personne conforte cette analyse.

(iii) La troisième fonction des PC factuels non expérientiels consiste à envisager de façon synthétique ou récapitulative les événements décrits dans

29 En moyen français, depuis n'a pas la même signification qu'en français moderne. Il peut n'indiquer que la postériorité et signifier « ensuite», «par la suite » (quand il est adverbe), ou " après ", "à la suite de », "à partir de " (quand il est préposition). L'intervalle temporel qu'il implique n'inclut donc pas nécessairement le repère temporel $\left(\mathrm{T}_{0}\right.$ pour le $\left.\mathrm{PC}\right)$. Depuis conjonction n'est pas, comme en français moderne, incompatible avec le PS. 
une séquence narrative antérieure. L'énoncé est alors factuel résomptif. Martin (1971) avait déjà relevé cet emploi du PC. En voici quelques exemples caractéristiques :

(47) [...] depuis le temps que je veins en son service, jusques à l'heure de son trespas, où j'estoye present, AY FAICT PLUS CONTINUELLE RÉSIDENCE AVEC LUY QUE NUL AUTRE [...] (Comm., Prologue)

[depuis le moment où j'entrai à son service jusqu'à l'heure de son trépas, auquel j'ai assisté, JE L'AI CÔTOYÉ DE FAÇON PLUS CONTINUE QUE NULLE AUTRE PERSONNE.]

(41) Je ne luy ouy oncques dire qu'il fust las ny ne luy vey jamais faire semblant d'avoir paour, ET SY AY ESTÉ SEPT ANNÉES DE RENG EN LA GUERRE AVECQUES LUY [...]. (Comm. I, 4)

[Jamais je ne l'entendis dire qu'il était las ni ne le vis laisser paraître qu'il avait peur, ET POURTANT J'AI ÉTÉ SEPT ANS DE SUITE À LA GUERRE AVEC LUI.]

(48) [Description de Louis XI, avec des IMP] Et ces termes et façons qu'il tenoit, dont j'ay parlé icy dessus, LUY ONT SAULVÉ LA COURONNE, veu les ennemys qu'il s'estoit luy mesmes acquis à son advenement au royaulme. (Comm. I, 10)

Ces PC factuels résomptifs sont prédisposés à délimiter des séquences textuelles. On les rencontre par exemple comme déclencheurs de relations de discours de type élaboration ou justification (au sens de Mann \& Thompson 1988). L'extrait ci-après est une illustration de la relation d'élaboration.

(49) Ledict conte de Warvic soustenoit la maison d'Iort, et le duc de Sombresset la maison de Lanclastre. TANT ONT DURÉ CES GUERRES QUE TOUS CEULX DE LA MAISON DE WARVIC ET DE SOMBRESSET EN ONT EU LES TESTES TRANCHÉES OU MORS EN BATAILLE. Le roy Edouard fist mourir son frère, duc de Clarance, en une pippe de malvoisye, pour ce qu'il se vouloit faire roy, comme l'on disoit. [Suit une liste de massacres] (Comm. I, 7)

[Ledit conte de Warvic soutenait la maison d'Iort, et le duc de Sombresset la maison de Lanclastre. CES GUERRES ONT DURÉ SI LONGTEMPS QUE TOUS LES MEMBRES DES MAISONS DE WARVIC ET DE SOMBRESSET ONT FINI PAR AVOIR LA TÊTE TRANCHÉE OU SONT MORTS AU COMBAT. Le roi Edouard fit mourir son frère, le duc de Clarance, dans une barrique de malvoisie, parce qu'il voulait être roi, disait-on.] Suit une liste de massacres.

L'énoncé en petites capitales, au PC, annonce et chapeaute une énumération de divers massacres rapportés au PS.

Le codage de la fonction de commentaire que réalise le PC, en contraste avec le PS narratif, n'est pas complètement rigide, comme en témoigne 1'extrait suivant :

(50) Il avoit quatre cens cranequiniers que luy avoit presté le conte pallatin, gens fort bien montéz $[. .$.$] ; et avoit cinq cens Suysses à pied, QUI FURENT LES$ 


\begin{abstract}
PREMIERS QUE ON VEIST EN CE ROYAULME ET ONT ESTÉ CEULX QUI ONT DONNÉ LE BRUYT AUX AULTRES QUI SONT VENUZ DEPUIS [...]. (Comm. I, 6)

[Il avait quatre cents arbalétriers que lui avait prêtés le conte palatin, hommes pourvus de très bonnes montures [...] ; il avait également cinq cents fantassins suisses, QUI FURENT LES PREMIERS QUE L'ON VITT DANS CE ROYAUME ET ONT ÉTÉ CEUX QUI ONT INFORMÉ LES AUTRES QUI SONT VENUS ENSUITE.]
\end{abstract}

Le commentaire porte sur les fantassins suisses et débute par une relative. Pourtant celle-ci comporte un PS (qui furent les premiers), et elle inclut ellemême une autre relative comportant elle aussi un PS (qu'on vit dans ce royaume). Mais la continuation de la première relative passe ensuite, elle, au PC (et ont été ceux qui...). On observe donc que dans cet extrait, tout se passe comme si le temps verbal, en tant qu'indice du registre narratif, marquait tout d'abord une certaine inertie par rapport à l'évolution du contenu du texte, pour s'accorder ensuite à ce nouveau contenu et le marquer grammaticalement comme un registre différent (par une sorte d'effet retard). Compte tenu des observations faites plus haut à propos de depuis, il est vraisemblable que la présence de cet adverbe à la fin de cet extrait a joué un certain rôle dans le passage au PC.

Cette transition PS > PC peut être corrélée à la durée des situations désignées, et engendrer ainsi un certain flottement entre le registre narratif et le registre factuel. C'est ce que suggère l'exemple ci-dessous :

(51) Toutesfois peu DURA ceste amytié, mais A DURÉ guerre entre le roy et le roy d'Arragon plus de seize ans et encores dure ce differant. (Comm. II, 8)

Il est intéressant de constater ici que le PS est associé à un état dont il est dit explicitement qu'il a été de courte durée, tandis que le PC est associé à un état dont il est dit qu'il dure encore à $\mathrm{T}_{0}$.

\title{
5.2. Énoncés factuels expérientiels
}

L'emploi expérientiel du PC est tout à fait commun dans le texte de Commynes. En voici deux exemples, où on retrouve d'ailleurs nos verbes du témoignage :

[...] J'AY VEU beaucoup de tromperies en ce monde [...]. (Comm. I, 10)

(53) En luy et tous autres princes que J'AY CONGNEUZ ou SERVY, AY CONGNEU du bien et du mal, car ilz sont hommes comme nous. (Comm., Prologue) [Chez lui comme chez tous les autres princes que J'AI CONNUS ou SERVIS, J'AI CONSTATÉ qu'il y avait du bien et du mal, car ils sont hommes comme nous.]

Cet emploi du PC n'a rien de surprenant en moyen français. On le rencontre en ancien français également : 
« En ma maisun ad une caitive franche,

Tant AD OÏT e sermuns e essamples,

Creire voelt Deu, chrestientet demandet. » (Ch. de Roland, CCXCVI)

["Dans ma maison, il y a une prisonnière de noble race; elle A ENTENDU tellement de sermons et de bons exemples qu'elle veut croire en Dieu et demande chrétienté ».]

Comme en français moderne, il peut arriver, que certaines formulations prêtent à une double interprétation. Ainsi, l'énoncé par lequel débute l'extrait ci-dessous pourrait théoriquement être interprété soit comme épisodique («ce jour-là je les ai vus tenir trois conseils dans une chambre...»), soit comme expérientiel ("il m'est arrivé de les voir tenir trois conseils dans une chambre... »). Toutefois la suite du texte (et je vis un jour que..., cela s'était déjà produit deux fois...) paraît indiquer que c'est la lecture expérientielle qui s'impose ici.

$$
\begin{aligned}
& \text { JE LES AY VEU TENIR TROIS CONSEILZ EN UNE CHAMBRE OÙ ILZ ESTOIENT TOUS } \\
& \text { ASSEMBLÉZ, et veiz ung jour qu'il en despleut bien au conte de Charroloys, } \\
& \text { car il s'estoit desja faict deux fois en sa presence [...]. (Comm. I, 12) } \\
& \text { [JE LES AI VUS TENIR TROIS CONSEILS DANS UNE PIÉCE OÙ ILS S'ÉTAIENT TOUS } \\
& \text { ASSEMBLÉS, et je vis un jour que cela déplut beaucoup au conte de } \\
& \text { Charrolois, car cela s'était déjà produit deux fois en sa présence.] }
\end{aligned}
$$

Nous avons signalé plus haut la forte affinité qu'il y a entre les énoncés d'expérience et les adverbiaux quantifieurs de fréquence et d'occurrences. Voici deux exemples de cooccurrence entre ces adverbiaux et le PC.

(56) Et telz seigneurs y a qui n'ont treze livres de rente en argent, qui se glorifient de dire: «Parlez à mes gens», cuydant par ceste parolle contrefaire les très grans. Aussi AY-JE BIEN VEU SOUVENT leurs serviteurs faire leur prouffit et leur donner à congnoistre qu'ilz estoient bestes. (Comm. I, 10)

IIl y a des seigneurs qui n'ont pas treize livres de rente en argent, qui se glorifient de dire: "Parlez à mes gens", pensant ainsi passer pour des personnes très importantes. C'est pourquoi J'AI VU BIEN SOUVENT leurs serviteurs tirer avantage de cette situation et leur faire savoir qu'ils étaient stupides.]

JE N'AY JAMAIS VEU tant tyrer pour peu de jours [...]. (Comm. I, 9)

[Je n'ai jamais vu autant tirer en si peu de jours.]

Dans ses rapports avec les énoncés d'expérience, l'adverbe jamais nous a paru particulièrement intéressant. Nous allons maintenant examiner plus en détail le cas de cet adverbe. 


\subsection{Jamais}

Nous avons vu qu'une des propriétés des énoncés d'expérience est d'impliquer un intervalle de validation, intervalle dont la borne terminale coïncide, en français moderne, avec $\mathrm{T}_{0}$ pour le $\mathrm{PC}$, mais qui se situe dans l'époque passée pour le PQP et dans l'époque future pour le FA. Par ailleurs l'adverbe jamais, quelle que soit sa lecture, implique nécessairement un intervalle temporel. Comment se présente cet intervalle dans les énoncés comportant cet adverbe, sachant qu'en moyen français, PC et PS n'occupent pas les mêmes territoires fonctionnels qu'aujourd'hui ?

\subsubsection{Jamais + PS, jamais + PC}

En fait, deux cas peuvent se présenter, sans d'ailleurs qu'on puisse toujours trancher avec certitude entre les deux. Le premier implique systématiquement le PS ; le second se rencontre aussi bien avec le PS qu'avec le PC.

Dans le premier cas, la borne terminale de l'intervalle impliqué par jamais se situe dans le passé. L'intervalle parcouru par l'adverbe est donc, pour des raisons propres au contenu même de l'énoncé et à son contexte, entièrement localisé dans le passé :

Car JAMAIS nous n'eusmes faulte de vivres [...]. (Comm. I, 7)

(59) Ny ne vey JAMAIS une seulle journée qu'il n'y eust escarmouche, quelque petit que ce fust; (Comm. I, 11)

[Et je ne vis jamais une seule journée où il n'y eut une escarmouche, ne serait-ce que bénigne.]

Il faut savoir que ces deux formulations sont chacune extraites d'un épisode narratif, dont elles constituent une sorte de commentaire rétrospectif. Dans les deux exemples, ce commentaire demeure à l'intérieur du cadre temporel de la narration: dans (58), l'absence de manque de nourriture concerne spécifiquement la durée de l'épisode narratif dont il a été question antérieurement; de même, l'absence de journée sans escarmouche évoquée dans (59) ne concerne, elle aussi, qu'un épisode narratif temporellement circonscrit. Dans ces deux exemples, l'intervalle que parcourt l'adverbe jamais est donc temporellement coextensif de l'épisode narratif commenté. Pour cette raison, on peut considérer ici que l'énoncé comportant jamais est une partie intégrante de la narration. Le fait qu'il y ait toujours, dans ce cas, un PS, en est une sorte de confirmation.

Dans le second cas, la borne terminale de l'intervalle impliqué par jamais coïncide avec $\mathrm{T}_{0}$. On rencontre alors aussi bien le PS que le PC.

(60) [...] et tant osé-je bien dire de luy, à son loz, qu'il ne me semble pas que JAMAIS j'aye congneu nul prince où il y eust moins de vices que en luy, à 
regarder le tout. (Comm. Prologue)

[et j'ose dire de lui, en son honneur, qu'il ne me semble pas que j'aie JAMAIS connu aucun prince en qui il y eût moins de vices que lui, tout bien considéré.]

(61) JAMAIS ne se mena traicté entre les François et Angloys, que le sens des François et leur habilité ne se monstrast par dessus celle des Angloys. (Comm. III, 8)

[JAMAIS il n'y eut négociation d'un traité entre Français et Anglais, sans que le savoir-faire des Français et leur habileté ne se montrassent supérieurs à ceux des Anglais.]

L'énoncé comportant jamais quitte ici le cadre temporel de la narration. Cet élargissement de la temporalité est particulièrement claire dans (60), où c'est l'ensemble des princes que le scripteur a connus durant son existence qui est envisagé : jamais est corrélé dans cet exemple à une opération de généralisation. Le second exemple pourrait à la rigueur être interprété avec une borne terminale située dans le passé ; l'énoncé ne serait alors valide que pour un intervalle limité ne sortant pas du cadre de la narration, comme dans (58) et (59). Mais cette interprétation est peu vraisemblable : le contexte d'où est extraite cette phrase (trop long à reproduire ici) indique que le propos doit être compris au sens le plus général, et reflète la connaissance, l' « expérience » qu'a le chroniqueur Commynes du savoir-faire des Français et des Anglais en matière de négociation de traités.

\subsubsection{Jamais + PS}

Il faut donc distinguer deux emplois de jamais + PS : l'un délimitant un intervalle entièrement localisé dans le passé, l'autre délimitant un intervalle qui court jusqu'à $\mathrm{T}_{0}$. On est donc maintenant fondé à considérer que quand la borne terminale de jamais se situe dans le passé, comme dans (58) et (59), l'énoncé n'est pas expérientiel et doit être considéré comme une variante d'énoncé narratif. En revanche, quand la borne terminale de jamais court jusqu'à $\mathrm{T}_{0}$, comme dans (60) et (61), l'énoncé est expérientiel (du moins il accepte cette lecture), et ceci indépendamment du temps verbal utilisé (PS ou $\mathrm{PC})$.

Bien évidemment, certaines occurrences sont difficilement décidables. Depuis, dont on a vu qu'il peut signifier, en moyen français, aussi bien « ensuite » que « depuis », est une cause fréquente d'ambiguïtés à cet égard, précisément en raison de cette polysémie. Dans la première acception, en effet, le tandem jamais depuis délimite un intervalle dont la borne terminale se situe dans le passé ; mais dans la seconde acception, cette borne terminale s'étend jusqu'à $\mathrm{T}_{0}$. Il faut donc avoir recours à des indices contextuels pour sélectionner l'une des deux interprétations. L'exemple suivant illustre bien cette ambiguïté : 
Ce détour par l'adverbe jamais confirme que dans le texte de Commynes, le PS se rencontre régulièrement dans des énoncés factuels expérientiels. Cette propriété tient au fait que ce temps verbal peut, en moyen français, étendre son champ d'action jusqu'à $\mathrm{T}_{0}$, comme le notait Martin. Compte tenu du fait qu'en français moderne, les énoncés expérientiels sont l'apanage des temps composés, on a là un domaine particulièrement intéressant pour observer l'évolution des rapports complexes, compétitifs, qu'entretiennent PS et PC dans cette période de l'histoire du français.

\subsubsection{Jamais et l'expression de la superlativité et de l'exceptionnalité}

On se bornera ici à poser quelques jalons à partir du texte de Commynes. Trois observations guideront notre analyse.

$1^{\text {ère }}$ observation. - A comparer quantitativement les occurrences de jamais + PS et jamais + PC, on s'aperçoit que la configuration jamais + PC est relativement rare. Dans notre corpus on en dénombre 6 occurrences, tandis que la configuration jamais + PS apparaît 42 fois.

$2^{\mathrm{e}}$ observation. - Avec le PC comme avec le PS, la majorité des occurrences de jamais concernent des énoncés dont la fonction est d'exprimer la superlativité ou l'idée d'exception.

$3^{\mathrm{e}}$ observation. - La configuration jamais $+\mathrm{PC}$ ne se rencontre que dans des énoncés où il s'agit d'exprimer la superlativité ou de l'idée d'exception.

Ces résultats sont résumés dans le Tableau 2.

\begin{tabular}{|l|c|c|c|c|}
\hline & \multicolumn{2}{|c|}{ jamais + PS } & \multicolumn{2}{c|}{ jamais + PC } \\
\hline & Expérientiel & Narratif & Expérientiel & Narratif \\
\hline $\begin{array}{l}\text { Superlativité- } \\
\text { exceptionnalité }\end{array}$ & 25 & 3 & 5 & 0 \\
\hline Autres & 8 & 6 & 1 & 0 \\
\hline
\end{tabular}

Tableau 2.- Nombre d'occurrences des configurations jamais + PS et jamais + PC dans les Livres I à III des Mémoires de P. de Commynes

\section{La superlativité et l'exceptionnalité}

Arrêtons-nous un instant sur ce que désignent ici les termes de « superlativité » et d' " exception». Dans notre corpus, la configuration jamais + PC/PS apparaît dans les contextes suivants : 
(i) Dans des énoncés négatifs ou jamais signifie simplement « aucune fois » :

(63) Je ne luy ouy oncques dire qu'il fust las ny NE luy VEY JAMAIS faire semblant d'avoir paour [...]. (Comm. I, 4)

[Jamais je ne l'entendis dire qu'il étais las ni ne le vis jamais laisser paraître qu'il avait peur.]

(ii) Dans des énoncés négatifs ou non négatifs, associés à l'expression de la superlativité ou de l'idée d'exception. On entend par là des formulations comme les suivantes :

(64) Nul homme NE PRESTA JAMAIS tant l'oreille aux gens ny ne s'enquist de tant de choses comme il faisoit [...]. (Comm. I, 10)

(65) Et entre tous ceulx que j'AY JAMAIS CONGNEU, le plus saige pour soy tyrer d'un mauvais pas en temps d'adversité, c'estoit le roy Loys unziesme [...]. (Comm. I, 9)

(66) [...] arrivèrent les ducs de Berry et de Bretaigne, que JAMAIS NE VEY arméz que ce jour. (Comm. I, 11)

Les exemples (64)-(65) illustrent ce que nous entendons par « expression de la superlativité »; les marqueurs en sont ici tant et le plus. (66) illustre ce que nous entendons par « expression de l'idée d'exception »; le marqueur est ici ne...que ce jour). Quand l'énoncé n'est pas négatif, comme dans (65), jamais est privé de ne et est une sorte d'indéfini signalant un intervalle de validation. Cet intervalle pourrait être formulé, pour cet exemple, par l'expression « au cours de ma vie et jusqu'à maintenant ».

(iii) Dans des énoncés non négatifs, mais hors contextes de superlativité ou d'exceptionnalité.

[...] il fault noter que tous les hommes qui JAMAIS ONT ESTÉ grans et FAICT grand chose ont commancé fort jeunes [...]. (Comm. I, 10)

[il faut noter que tous les hommes qui ONT JAMAIS ÉTÉ grands et ONT RÉALISÉ de grandes choses ont commencé fort jeunes.]

\section{L'hypothèse du figement}

Il est remarquable que parmi ces trois emplois, ceux qui sont associés à l'expression de la superlativité ou de l'idée d'exception soient de loin les plus fréquents. Le Tableau 2 ci-dessus montre que nos données totalisent 48 occurrences de jamais + PC/PS : sur ces 48 occurrences, 33 sont associées à la superlativité-exceptionnalité. Ces chiffres suggèrent qu'il existe un lien étroit entre l'adverbe jamais et l'expression de la superlativité ou de l'exception, tout particulièrement avec le PS. Plus exactement, ils suggèrent que la configuration jamais + PS est une formulation plus ou moins figée, à 
caractère phraséologique, lorsqu'elle intervient dans l'expression de la superlativité ou de l'idée d'exception.

Une autre observation vient conforter cette analyse. C'est le fait que dans certains cas, l'adverbe jamais voit sa composante temporelle s'éroder. L'adverbe tend alors à devenir un simple intensificateur de la modalité réfutative ou assertive de l'énoncé. Cette tendance apparaît dans des exemples comme les suivants :

$$
\begin{aligned}
& \text { JAMAIS plus grant fuytte NE FUT des deux costéz. (Comm. I, 4) } \\
& \text { [JAMAIS IL N'Y EUT plus grande débandade dans les deux camps] }
\end{aligned}
$$

Ledict de Vaneloc ne me dist que parolles honnestes et quelque peu d'excuse en la faveur dudict conte, son cappitaine, et les biens qu'il luy avoit faitz. Mais quant aux autres, qui estoient avecques luy, JAMAIS NE FURENT SI DESBORDÉZ, car ceulx que je pensoye les meilleurs pour ledit roy estoient ceulx qui plus le menassoient, [...]. (Comm. III, 6)

[... Quant aux autres, qui étaient avec lui, JAMAIS ILS NE FURENT À CE POINT DÉCHAÎNÉS, car ceux que je croyais les plus favorables audit roi étaient ceux qui lui étaient les plus hostiles]

Dans ces exemples, jamais peut certes être interprété littéralement, c'est-àdire temporellement. Mais le contexte discursif et le sens même suggèrent ici une lecture un peu différente. De fait, l'assertion consistant à dire que la situation décrite (i.e. une certaine débandade, dans (68), la tenue de certains propos, dans (69)) n'est jamais advenue dans le passé, cette assertion est avant tout au service de l'expression d'une valeur superlative. Ces deux formulations sont au fond une manière de dire, respectivement: «la débandade fut extrême dans les deux camps » et « ils furent complètement déchainés $»^{30}$.

D'autres indices confirment que la configuration jamais + PS, de loin la plus fréquente dans l'emploi qui nous intéresse ici, présente des symptômes de figement. On note par exemple, dans cet emploi, une présence quasi systématique de la $1^{\text {ère }}$ personne; on observe également que les verbes appelés plus haut "verbes du témoignage » (principalement voir, connaître, entendre) y sont omniprésents. Voici un petit échantillon de formulations illustrant ce qui précède :

(70) a. de ce petit que j'ay veu N'EN VIZ JAMAIS qui eussent meilleur voulloir de combattre (Comm. I, 3)

b. JE NE VEY JAMAIS si belle compaignie (Comm. I, 6)

30 En français moderne, la tendance de jamais à fonctionner comme un marqueur d'intensité est pratiquement aboutie dans des formulations comme : Il est trop tard, jamais je n'attraperai mon train, où on ne peut plus interpréter jamais comme signifiant « aucune fois dans l'intervalle temporel considéré ». Pour des observations analogues sur son équivalent anglais never, voir Cheshire (1998). 
c. le plus pouvre roy, habandonné de ses serviteurs, que JE VEÏZ JAMAIS. (Comm. II, 8)

d. ne luy VËYZ JAMAIS tant de gens ensemble (Comm. II, 2)

e. Excepté ce jour, ne CONGNEU JAMAIS que on eust esperance de combattre (Comm. I, 11)

f. ung des plus saiges chevaliers et des plus entenduz que je CONGNEÜ JAMAIS (Comm. II, 2)

g. a myeulx sceü entendre cest art de separer les gens que nul autre prince que JAMAIS JE CONGNEÜ (Comm. II, 1)

h. JAMAIS JE NE CONGNEÜ si saige homme en adversitéz (Comm. III, 12)

i. JAMAIS J'AYE CONGNEU nul prince où il y eust moins de vices que en luy (Comm. I, Prologue)

Ces éléments suggèrent que, lorsqu'il s'agit d'exprimer la superlativité, ou simplement d'attribuer une propriété quelconque, par exemple à un personnage ou à une situation, en insistant sur le haut degré de cette propriété, Commynes cède à une sorte d'automatisme langagier consistant à formuler ce contenu dans le moule d'un énoncé d'expérience. D'où : parcours fictif d'un certain intervalle temporel (indiqué par jamais) et assertion que cette propriété ne se rencontre pas à un pareil degré dans cet intervalle. Le procédé est clairement hyperbolique. L'utilisation qui y est faite de la structure existentielle et aspectuo-temporelle des énoncés d'expérience relève elle-même de la figure. Au total, le sens de l'énoncé n'est pas vraiment compositionnel : il y a «construction», au sens donné à ce terme dans les grammaires du même nom. Le fait que la structure de l'énoncé d'expérience devienne un moyen rhétorique de la superlativité ne peut que favoriser l'érosion sémantique de jamais, érosion qui se manifeste par une tendance à fonctionner comme un simple marqueur d'intensité dépourvu de signification temporelle.

Quel enseignement tirer de ces analyses, concernant les rapports entre PC et PS ? C'est que la configuration jamais + PS, en raison de son caractère partiellement automatique, partiellement figé, a très vraisemblablement fonctionné comme un environnement «conservateur» pour le PS. Les observations qui précèdent portent à penser que cette configuration, quand elle est destinée à exprimer la superlativité, est à cette époque partiellement fossilisée ${ }^{31}$.

31 Quelque 50 ans après Commynes, dans les nouvelles de l'Heptaméron de Marguerite de Navarre, des formules comme : le plus malheureux qui oncques fut, les meilleures viandes que je mangeai jamais, les plus fausses opinions que je vis jamais, la plus honnête amour dont on oüt jamais parler, etc., sont pratiquement des tics de langage. On en trouve jusque dans les dialogues, alors que de façon générale les PS sont rares dans les dialogues de l'Heptaméron (ce qui est un indice supplémentaire de figement). Il est intéressant d'observer que ces formulations, qui sont à strictement parler extra-narratives, apparaissent la 
Une autre raison peut expliquer la tendance au maintien du PS dans ces contextes. Si l'on admet que l'utilisation de la structure expérientielle dans ces énoncés est, pour une part au moins, figurale, rhétorique, alors les formulations concernées pourraient être analysées comme l'amalgame de deux propositions. Soit l'exemple suivant :

Jamais je ne fus si étonné.

Dans la perspective exposée ci-dessus, cet énoncé pourrait être considéré comme résultant de 1'amalgame des deux propositions P1 et P2 :

P1 : « je fus \{très / passablement / extrêmement... \} étonné »

P2 : «jamais je n'ai été \{ainsi / autant...\} étonné »

La valeur proprement superlative est exprimée par P1, la valeur expérientielle par P2. Or, on notera que le temps verbal de P1 est un PS, et que P1 pourrait tout à fait figurer avec le statut d'événement dans le cours d'une narration. Tout autre est P2, qui traite l'événement en question comme un type et parcourt un intervalle temporel courant jusqu'à $T_{0}$ (pour asserter que ce type d'événement ne s'y trouve pas, ne s'y est jamais produit). On retrouve très exactement ici notre définition des énoncés d'expérience (cf. section 3.2.2.). Ce que montre cette décomposition sémantique, c'est que (71) amalgame un énoncé narratif et un énoncé d'expérience. Si l'on admet que cet exemple peut être décomposé ainsi, alors la tendance du PS à se maintenir dans ces structures trouve ici une explication supplémentaire.

\section{Bilan et épilogue}

Nous espérons avoir montré que le PC, quand il est employé comme temps processif, c'est-à-dire pour désigner une situation passée en vertu du fait que son évocation présente une certaine pertinence à $\mathrm{T}_{0}$, se caractérise entre autres par les propriétés suivantes : d'une part, il produit un effet de saillance plus ou moins fort sur la phase événementielle, processive, de la situation, phase qu'il met au premier plan pour ce qui est de la dimension informationnelle et référentielle du discours ; mais d'autre part, il entretient un lien complexe avec le moment de l'énonciation. Il est frappant de constater que dans les travaux sur le $\mathrm{PC}$, ce lien est régulièrement mentionné, mais jamais vraiment analysé, ni à fortiori problématisé. En somme, tout se passe comme si le PC utilisé comme temps du passé, ne faisant que déplacer le centre d'attention de la phase résultative à la phase processive, conservait à

plupart du temps dans des narrations ; elles relèvent du commentaire stéréotypé de certains épisodes narratifs. C'est vraisemblablement la raison pour laquelle elles sont fréquentes dans les Mémoires de Commynes, texte où la narration occupe une place importante. 
titre de résidu sémantique une vague mémoire de l'état résultant qu'il désigne dans ses emplois résultatifs. Or, cette conception de la résultativité du PC processif, c'est-à-dire de son lien avec $\mathrm{T}_{0}$, est contredite par de nombreuses données, et laisse complètement dans l'ombre les conséquences épistémiques qui sont associées à l'emploi de ce temps verbal. La thèse que nous avons tenté de défendre est que le PC processif sert fondamentalement à dire l'effectivité de l'advenue d'une situation. Plus exactement, il sert à asserter que l'advenue de la situation qu'il désigne est, du point de vue de l'énonciateur, considérée comme valide à $\mathrm{T}_{0}$. Cette conception renvoie à la notion de « fait» ou d'énoncé «factuel». Une situation n'est un fait que si elle est reconnue par un sujet de conscience (qui la valide comme advenue) relativement à un repère temporel (le moment où elle est validée comme un fait). Il résulte de cette définition qu'un fait nouveau n'est pas une situation qui vient de se produire, mais une situation qui vient de parvenir à la connaissance d'un sujet de conscience.

Le PC employé comme temps du passé est souvent décrit comme impliquant un point de vue localisé à $\mathrm{T}_{0}$, ou comme indiquant que l'événement désigné, bien que passé, présente une certaine actualité. L'analyse qui vient d'être résumée nous semble donner une certaine consistance à cette idée somme toute extrêmement vague de point de vue ou d'actualité.

En second lieu, nous avons développé l'idée selon laquelle la factualité telle qu'elle s'exprime dans le PC, et plus généralement dans les temps composés, se décline en plusieurs variantes, appelées ici "épisodique », « résomptive » et « expérientielle». Cette dernière concerne ce qu'on appelle le «parfait d'expérience» ou «parfait existentiel». Nous en avons donné une description relativement détaillée, en insistant sur un certain nombre d'éléments qui lui sont fréquemment associés : adverbiaux quantifieurs de fréquence ou d'occurrences, référence indéfinie, verbes du témoignage (voir, entendre, connaître) à la $1^{\text {ère }}$ personne, expression désignant un intervalle de validation, etc. Du point de vue de leur insertion dans le discours, il apparaît que les énoncés d'expérience trouvent leur milieu le plus naturel dans le genre argumentatif.

En troisième lieu, nous avons estimé important de revenir sur le concept de narration. Il nous a semblé, en effet, que dans les descriptions qui sont données du PC, ce concept est parfois utilisé de manière trop extensive, étant pratiquement identifié à la référence à un événement passé : tout $\mathrm{PC}$ utilisé de façon processive, référant à une situation passée localisée dans un intervalle n'incluant pas $\mathrm{T}_{0}$, serait ipso facto narratif. Le fait d'accepter un complément adverbial localisant la situation dans un intervalle dont la borne terminale est antérieure à $\mathrm{T}_{0}$ (caractéristique du PC qui s'est installée à partir du XVIII ${ }^{\mathrm{e}} \mathrm{s}$.) est certes une condition nécessaire à un emploi narratif de ce temps verbal, mais certainement pas une condition suffisante. Ce point nous paraît 
important, tout particulièrement dans les discussions concernant le rapport entre PC et valeur d'aoriste.

Enfin, dans la section proprement analytique de cet article, nous avons entrepris d'explorer un corpus de moyen français (Livres I-III des Mémoires de Commynes) afin d'étudier, en utilisant les analyses et concepts présentés dans les sections précédentes, les emplois qui y étaient faits du PC processif et du PS. Le but était principalement de mieux comprendre le rôle de la factualité, et en particulier des énoncés d'expérience, dans l'évolution du PC et de ses rapports avec le PS. Les principales observations sont les suivantes :

1. Le PC processif, dans le texte étudié, est toujours factuel et intervient dans des énoncés extrêmement typés. Quand il n'est pas expérientiel, on le rencontre avec une fréquence élevée dans des énoncés comportant des éléments de déixis textuelle. Il est alors associé à un fonctionnement métatextuel, c'est-à-dire à des séquences dont la fonction est de baliser le texte afin de permettre au lecteur de s'y orienter plus aisément. On le rencontre également souvent dans des commentaires jalonnant le flux narratif. Il permet alors d'apporter une information annexe concernant par exemple un protagoniste, de formuler un jugement, ou encore d'anticiper sur le devenir de la narration. Dans tous ces cas, le texte quitte la temporalité narrative pour passer à un registre où la relation avec le lecteur redevient pertinente et où l'argumentatif peut prendre place. Les énoncés factuels de type résomptifs jouent ici un rôle important.

2. Nous n'avons rencontré, dans notre corpus, aucun exemple de séquence de PC qui pourrait être caractérisée de narrative.

3. On rencontre en revanche des PC dans des énoncés expérientiels (parfait d'expérience). Cet emploi paraît bien établi, en ancien comme en moyen français. On observe alors, comme en français moderne, les mêmes affinités avec, par exemple, les adverbiaux quantifieurs de fréquence ou d'occurrences.

4. Cependant, nous avons constaté que pour la formulation de certains types d'énoncés d'expérience, PC et PS entrent en concurrence. Il s'agit d'énoncés comportant l'adverbe jamais. Cet adverbe, dont le sens implique un intervalle temporel, apparaît dans deux types de contextes quand il porte sur un PS : d'une part dans des énoncés qui ne sortent pas du cadre temporel de la narration, et dont l'intervalle temporel est par conséquent entièrement localisé dans le passé; d'autre part, dans des énoncés dont l'intervalle temporel s'étend - du moins peut s'étendre - jusqu'à $\mathrm{T}_{0}$. Par définition, seuls ces derniers sont interprétables comme des énoncés d'expérience. Or, ces énoncés présentent eux-mêmes une caractéristique particulière : ce sont très majoritairement des formulations dans lesquelles est exprimée l'idée de superlativité (type : jamais je ne fus si étonné) ou d'exception. Le nombre important de ces formulations, de même que d'autres indices que nous avons mis en évidence, indiquent qu'il s'agit d'automatismes de l'expression, de 
formulations partiellement figées. Cela signifie que l'utilisation qui y est faite de la structure expérientielle n'est au fond qu'un moyen rhétorique au service de la superlativité. Or, dans ces constructions, le PS est considérablement plus fréquent que le PC. L'hypothèse que l'on peut dès lors formuler est que c'est précisément ce caractère phraséologique qui explique le maintien du PS dans ces structures, alors même que le PC paraît bien installé comme grammème de la valeur d'expérience. Les constructions exprimant la superlativité au moyen de la configuration jamais + PS fonctionnent ainsi comme des niches protectrices pour le PS.

On terminera cet épilogue par deux remarques. La première concerne la question de l'aoriste ; la seconde, celle des genres textuels.

La question de savoir si le PC est ou non devenu un aoriste préoccupe les linguistes depuis longtemps. Mais il s'agit d'abord de s'entendre sur ce qu'on appelle « aoriste». Si, par ce terme, on désigne une valeur aspectuotemporelle caractérisée par (i) la perfectivité, c'est-à-dire la prise en compte de l'intégralité du procès, de sa borne initiale à sa borne terminale, et (ii) par l'absence de tout rapport avec l'énonciation (autrement dit, avec le repère temporel $\mathrm{T}_{0}$, avec un énonciateur sujet de conscience et avec la relation d'interlocution), alors il nous faut conclure que, s'il est en effet douteux que le PC soit devenu un aoriste au sens plein du terme, il est en revanche absolument certain que le PS, lui, a complètement achevé son processus d'aoristisation. Il suffit pour s'en convaincre de considérer quelle était la panoplie de ses emplois en moyen français. En d'autres termes, alors que plusieurs observations indiquent que la dérive aoristique du PC est loin d'être achevée, tout indique que le repli aoristique du PS est, lui, définitivement consommé.

Mis à part la narration et l'argumentation, il a fort peu été question des genres textuels dans cette étude. On devrait pourtant toujours avoir, en arrière-plan de toute réflexion sur les temps verbaux, la question des genres de texte. Cela est tout particulièrement vrai quand il s'agit de diachronie. Or, un point qu'on oublie souvent est que les genres textuels ont eux-mêmes une histoire, et que cette histoire entretient des rapports complexes avec celle des formes linguistiques. Combettes (2014) observe que la période du moyen français, précisément, coïncide avec un développement important du genre argumentatif en langue française - auparavant les textes les plus représentatifs de ce genre étaient rédigés en latin. Selon cet auteur, cette évolution soulève plusieurs questions. On peut par exemple se demander quelle a été l'influence de ce "nouveau » genre sur les formes linguistiques en français. On peut également se demander dans quelle mesure ce changement de langue, pour le genre argumentatif, ne s'est pas accompagné d'un transfert de pratiques rédactionnelles du latin au français. Cette seconde question, note Combettes, est d'autant plus pertinente que les auteurs de cette époque étaient dans une situation de quasi bilinguisme. 
Dans son ouvrage sur le moyen français, Martin (1971) fait part d'une observation qui montre bien l'importance des pratiques rédactionnelles et des genres dans la compréhension des faits grammaticaux. Il note que dans la première période du moyen français $\left(\mathrm{XIV}^{\mathrm{e}} \mathrm{s}\right.$.), le $\mathrm{PC}$ perd du terrain par rapport au PS, mais que dans la seconde période $\left(X^{\mathrm{e}} \mathrm{s}\right.$.), le $\mathrm{PC}$ regagne du terrain. L'explication de ce phénomène est la suivante. L'un des usages fréquents du PC en ancien français est d'exprimer un état dans le contexte d'une narration au présent historique; il a alors la valeur d'un présent résultatif. Or, on observe au cours du $\mathrm{XIV}^{\mathrm{e}} \mathrm{s}$., dans les pratiques narratives, une très sensible diminution de l'emploi du présent historique. C'est cette diminution qui a entraîné avec elle celle du PC, alors même que ce dernier, indépendamment de ce fait, se trouvait déjà engagé dans une phase d'expansion.

\section{Références}

Apothéloz, D. (2010a). Le passé surcomposé et la valeur de parfait existentiel, Journal of French Language Studies 20/2 : 105-126.

Apothéloz, D. (2010b). De l'usage argumentatif du parfait d'expérience, in : J. Górnikiewicz; H. Grzmil-Tylutki; I. Piechnik (éds), En quête de sens. Etudes dédiées à Marcela Światkowska. - W poszukiwaniu znaczeń. Studia dedykowane Marceli Świątkowskiej, Kraków : Wyd. UJ, 29-38.

Apothéloz, D. (2012). La concurrence du passé composé et du passé surcomposé dans l'expression de la valeur de parfait d'expérience, in : L. de Saussure; A. Rihs (éds), Etudes de sémantique et pragmatique françaises, Berne : Peter Lang, 39-65.

Apothéloz, D. (à paraître). Parfait existentiel et futur antérieur « de bilan », in : D. Roszko ; J. Satoła-Staśkowiak (éds), Semantyka a konfrontacja językowa, vol. 5, Warszawa : SOW.

Apothéloz, D. ; Combettes, B. (2011). Saillance et aspect verbal : le cas du plus-que-parfait, in : O. Inkova (éd.), Saillance. Aspects linguistiques et communicatifs de la mise en évidence dans un texte, Vol. 1, Besançon : Presses Universitaires de Franche-Comté, 225-246.

Apothéloz, D. ; Nowakowska, M. (2010). La résultativité et la valeur de parfait en français et en polonais, in: E. Moline ; C. Vetters (éds), Temps, aspect et modalité en français, Amsterdam: Rodopi, 1-23. (Cahiers Chronos 21)

Bache, C. ; Basbøll, H. ; Lindberg, C.-E. (eds), (1994). Tense, Aspect and Action. Empirical and Theoretical Contributions to Language Typology, Berlin : W. de Gruyter. 
Barbazan, M. (2007). Le trait [+/- allocutif]. Un principe explicatif de l'opposition du passé simple et du passé composé, Romanische Forschungen $119:$ 429-463.

Barceló, G.J.; Bres, J. (2006). Les temps de l'indicatif en français, Paris : Ophrys.

Benveniste, E. (1959). Les relations de temps dans le verbe français, Bulletin de la Société de Linguistique LIV/1. Repris dans Problèmes de linguistique générale, 1, Paris : Gallimard, 1966, 237-250.

Bres, J. (2010). Polysémie ou monosémie du passé composé ? Actualisation, interaction, effets de sens produits, in : N. Flaux; D. Stosic; C. Vet (éds), Interpréter les temps verbaux, Berne : Peter Lang, 161-180.

Brunot, F. ; Bruneau, C. (1949). Précis de grammaire historique de la langue française, Paris : Masson.

Buridant, C. (2000). Grammaire nouvelle de l'ancien français, Paris: SEDES.

Bybee, J. ; Perkins, R.; Pagliuca, W. (1994). The evolution of grammar. Tense, aspect and modality in the languages of the world, Chicago: The Chicago University Press.

Caron, P. ; Liu, Y.-C. (1999). Nouvelles données sur la concurrence du passé simple et du passé composé dans la littérature épistolaire, L'Information grammaticale $82: 38-50$.

Caudal, P. ; Vetters, C. (2007). Passé composé et passé simple : sémantique diachronique formelle, in: E. Labeau; C. Vetters; P. Caudal (éds), Sémantique et diachronie du système verbal français, Amsterdam: Rodopi, 121-151. (Cahiers Chronos 16)

Cheshire, J. (1998). English negation from an interactional perspective, in : I. Tieken-Boon van Ostade; G. Tottie ; W. van der Wurff (eds), Negation in the History of English, Berlin : W. de Gruyter, 29-54.

Combettes, B. (2014). L'émergence du texte argumentatif en français : type de texte et diachronie, in: M. Monte; G. Philippe (éds), Genres et textes. Déterminations, évolutions, confrontations, Lyon: Presses Universitaires de Lyon, 225-237.

Commynes, P. de (1924). Mémoires, tome 1, édité par J. Calmette et G. Durville, Paris : Les Belles Lettres.

Comrie, B. (1976). Aspect, Cambridge : Cambridge University Press.

Cornu, M. (1953). Les formes surcomposées en français, Berne: A. Francke.

Dahl, Ö. (1985). Tense and aspect systems, Oxford : Blackwell.

Dahl, Ö. (ed.), (2000). Tense and aspect in the languages of Europe, Berlin : W. de Gruyter.

Desclés, J.-P.; Guentchéva, Z. (2003). Comment déterminer les significations du passé composé par une exploration contextuelle, Langue française $138:$ 48-60. 
Ducrot, O. (1983). Opérateurs argumentatifs et visée argumentative, Cahiers de linguistique française $5:$ 7-36.

Estienne, H. (1569). Traicté de la conformité du langage françois avec le grec, Paris : Chez Jaques du Puis.

Flaux, N. ; Stosic, D ; Vet, C. (éds) (2010). Interpréter les temps verbaux, Berne : Peter Lang.

Fournier, N. (1998). Grammaire du français classique, Paris : Belin.

Franckel, J.-J. (1989). Etude de quelques marqueurs aspectuels du français, Genève : Droz.

Fryd, M. (1998). Present perfect et datation : une dérive aoristique ?, in : A. Borillo; C. Vetters; M. Vuillaume (éds), Regards sur l'aspect, Amsterdam : Rodopi, 29-50. (Cahiers Chronos 2)

Galet, Y. (1974). Illustration de la théorie des niveaux d'énonciation, Langue française $21: 26-42$.

Górnikiewicz, J. (2012). Le chien est sorti. Pies wyszedt / wychodził / byt... Mais finalement où est-il ? Quelques remarques sur l'expression de la résultativité en français et en polonais, Romanica Cracoviensia 12 : 90103.

Grize, J.-B. (1996). Logique naturelle et communications, Paris : Presses Universitaires de France.

Guentchéva, Z. (1990). Temps et aspect: l'exemple du bulgare contemporain, Paris : Editions du CNRS.

Guentchéva, Z. (1994). Manifestations de la catégorie du médiatif dans les temps du français, Langue française $102: 8-23$.

Guillaume, G. (1965). Temps et verbe. Théorie des aspects, des modes et des temps suivi de L'architectonique du temps dans les langues classiques, Paris : Honoré Champion.

Johanson, L. (2000). Viewpoint operators in European languages, in : Ö. Dahl (2000), 27-188.

Karolak, S. (2007). Składnia francuska o podstawach semantycznych [Syntaxe française à bases sémantiques], Tom 1, Kraków: Collegium Columbinum.

Karolak, S. (2008). Remarques sur l'équivalence du passé imperfectif polonais et des temps passés en français, Verbum XXX, $\mathrm{n}^{\circ} 2-3: 125$ 146.

Koschmieder, E. (1929). Zeitbezug und Sprache. Ein Beitrag zur Aspekt- und Tempusfrage, Leipzig, Berlin: B.G. Teubner. Trad. franç.: Les rapports temporels fondamentaux et leur expression linguistique. Contribution à la question de l'aspect et du temps, Villeneuve-d'Ascq : Presses Universitaires du Septentrion, 1996.

Kuryłowicz, J. (1977). Problèmes de linguistique indo-européenne, Wrocław, Warszawa, Kraków : Ossolineum. 
Labeau, E. ; Bres, J. (eds), (2014). Evolution in Romance verbal systems, Berne : Peter Lang.

Lambrecht, K. (1994). Information structure and sentence form, Cambridge : Cambridge University Press.

Leech, G.N. (1971). Meaning and the English Verb, London : Longman.

Luscher, J.-M.; Sthioul, B. (1996). Emplois et interprétations du passé composé, Cahiers de linguistique française 18 : 187-217.

Mann, W.C. ; Thompson, S.A. (1988). Rhetorical Structure Theory : Toward a functional theory of text organization, Text 8(3) : 243-281.

Marchello-Nizia, C. (1999). Le français en diachronie: douze siècles d'évolution, Paris : Ophrys.

Martin, R. (1971). Temps et aspect. Essai sur l'emploi des temps narratifs en moyen français, Paris : Klincksieck.

Maupas, C. (1625). Grammaire et syntaxe françoise, $2^{\mathrm{e}}$ édition, Paris : Adrian Bacot. $1^{\text {ère }}$ édition, Bloys : Philippes Cottereau, 1607.

McCawley, J.D. (1971). Tense and time reference in English, in : C.J. Fillmore ; D.T. Langendoen (dir.), Studies in Linguistic Semantics, New York : Holt, Rinehart and Winston, 96-113.

Mellet, S. (2000). Le parfait latin : un praeteritum perfectum, in : A. Carlier ; V. Lagae ; C. Benninger (éds), Passé et parfait, Amsterdam : Rodopi, 95-106. (Cahiers Chronos 6)

Michaelis, L.A. (1998). Aspectual grammar and past-time reference, London, New York : Routledge.

Mittwoch, A. (2008). The English Resultative perfect and its relationship to the Experiential perfect and the simple past tense, Linguistics and Philosophy 31 : 323-351.

$\mathrm{RCG}=$ Registres $d u$ Consistoire de Genève au temps de Calvin, Tome 3, Genève : Droz.

Rebotier, A. (2014). French participle agreement with avoir. Current trends as an indication of grammaticalization, in : E. Labeau; J. Bres (eds), (2014), 115-144.

Revaz, F. (1996). Passé simple et passé composé : entre langue et discours, Etudes de linguistique appliquée 102 : 175-190.

Schøsler, L. (1994). Did Aktionsart ever "compensate" verbal aspect in Old and Middle French ?, in : C. Bache ; H. Basbøll ; C.-E. Lindberg (eds), (1994), 165-184.

Schøsler, L. (2004). «Tu eps l'as deit» / «tut s'en vat declinant». Grammaticalisation et dégrammaticalisation dans le système verbal du français illustrées par deux évolutions, celle du passé composé et celle du progressif, Aemilianense 1 : 517-568.

de Swart, H. (2010). Circonstanciels temporels et aspect verbal : interaction dans les contextes épisodiques et habituels, in : N. Flaux ; D. Stosic ; C. Vet (éds), (2010), 83-106. 
Timberlake, A. (1977). Reanalysis and actualization in syntactic change, in : C. Li (ed.), Mechanisms of Syntactic Change, Austin (Texas) : University of Texas Press, 141-177.

Van de Velde, D. (2006). Grammaire des événements, Villeneuve d'Ascq : Presses Universitaires du Septentrion.

Vendler, Z. (1967). Facts and events, in: Z. Vendler, Linguistics in Philosophy, Ithaka (NY) : Cornell University Press, 122-146.

Vet, C. (1992). Le passé composé : contextes d'emploi et interprétation, Cahiers de Praxématique 19 : 37-59.

Vuillaume, M. (1990). Grammaire temporelle des récits, Paris : Minuit.

Waugh, L.R. (1987). Marking time with the passé composé : toward a theory of the perfect, Linguisticae Investigationes XI/1 : 1-47.

Weinrich, H. (1964). Tempus. Besprochene und erzählte Welt, Stuttgart: Kohlhammer Verlag. Trad. franç. : Le temps, Paris : Ed. du Seuil, 1973.

Wilmet, M. (1970). Le système de l'indicatif en moyen français, Genève : Droz.

Wilmet, M. (1997). Grammaire critique du français, Louvain-la-Neuve : Duculot. 